\title{
Structure of the Partition Function and Transfer Matrices for the Potts Model in a Magnetic Field on Lattice Strips
}

\author{
Shu-Chiuan Chang ${ }^{* *}$ and Robert Shrock ${ }^{\dagger}$ \\ (a) Department of Physics \\ National Cheng Kung University \\ Tainan 70101, Taiwan and \\ (b) C. N. Yang Institute for Theoretical Physics \\ State University of New York \\ Stony Brook, N. Y. 11794
}

\begin{abstract}
We determine the general structure of the partition function of the $q$-state Potts model in an external magnetic field, $Z(G, q, v, w)$ for arbitrary $q$, temperature variable $v$, and magnetic field variable $w$, on cyclic, Möbius, and free strip graphs $G$ of the square (sq), triangular (tri), and honeycomb (hc) lattices with width $L_{y}$ and arbitrarily great length $L_{x}$. For the cyclic case we prove that the partition function has the form $Z\left(\Lambda, L_{y} \times L_{x}, q, v, w\right)=\sum_{d=0}^{L_{y}} \tilde{c}^{(d)} \operatorname{Tr}\left[\left(T_{Z, \Lambda, L_{y}, d}\right)^{m}\right]$, where $\Lambda$ denotes the lattice type, $\tilde{c}^{(d)}$ are specified polynomials of degree $d$ in $q, T_{Z, \Lambda, L_{y}, d}$ is the corresponding transfer matrix, and $m=L_{x}\left(L_{x} / 2\right)$ for $\Lambda=s q$, tri $(h c)$, respectively. An analogous formula is given for Möbius strips, while only $T_{Z, \Lambda, L_{y}, d=0}$ appears for free strips. We exhibit a method for calculating $T_{Z, \Lambda, L_{y}, d}$ for arbitrary $L_{y}$ and give illustrative examples. Explicit results for arbitrary $L_{y}$ are presented for $T_{Z, \Lambda, L_{y}, d}$ with $d=L_{y}$ and $d=L_{y}-1$. We find very simple formulas for the determinant $\operatorname{det}\left(T_{Z, \Lambda, L_{y}, d}\right)$. We also give results for self-dual cyclic strips of the square lattice.
\end{abstract}

\section{INTRODUCTION}

The $q$-state Potts model has served as a valuable system for the study of phase transitions and critical phenomena [1]-[5], and recently there has been considerable interest in its connections with mathematical graph theory [6]-[8]. For two-dimensional lattices, additional insights into the critical behavior have been obtained from conformal algebra methods $[9,10]$. On a lattice, or, more generally, on a graph $G$, at temperature $T$ and in an external magnetic field $H$, the original Hamiltonian formulation of this model is defined by the partition function

$$
Z=\sum_{\left\{\sigma_{n}\right\}} e^{-\beta \mathcal{H}}
$$

with the Hamiltonian

$$
\mathcal{H}=-J \sum_{\langle i j\rangle} \delta_{\sigma_{i}, \sigma_{j}}-H \sum_{i} \delta_{\sigma_{i}, 1}
$$

where $\sigma_{i}=1, \ldots, q$ are classical spin variables on each vertex (site) $i \in G, \beta=\left(k_{B} T\right)^{-1}$, and $\langle i j\rangle$ denote pairs of adjacent vertices. The graph $G=G(V, E)$ is defined by its vertex (site) set $V$ and its edge (bond) set $E$; we denote the number of vertices of $G$ as $n=n(G)$ and the number of edges of $G$ as $e(G)$. Without loss of generality, we take $G$ to be connected and we take the magnetic field to single out the spin value $\sigma_{i}=1$. We use the notation

$$
K=\beta J, \quad h=\beta H, \quad y=e^{K}, \quad v=y-1, \quad w=e^{h}
$$

*Electronic address: scchang@mail.ncku.edu.tw

$\dagger^{\dagger}$ Electronic address: robert.shrock@stonybrook.edu 
Thus, the physical ranges of $v$ are $v \geq 0$ for the Potts ferromagnet, and $-1 \leq v \leq 0$ for Potts antiferromagnet. Positive $H$ gives a weighting that favors spin configurations in which spins have the value 1 , while negative $H$ disfavors such configurations. For positive and negative $H$, the physical range of $w$ is $w>1$ and $0 \leq w<1$, respectively.

The original definition of the Potts model, (1.1) and (1.2), requires $q$ to be in the set of positive integers $\mathbb{N}_{+}$. This restriction is removed for the zero-field Potts model by the Fortuin-Kasteleyn cluster representation 11.

$$
Z(G, q, v)=\sum_{G^{\prime} \subseteq G} v^{e\left(G^{\prime}\right)} q^{k\left(G^{\prime}\right)}
$$

where $G$ is an arbitrary graph, $G^{\prime}=\left(V, E^{\prime}\right)$ with $E^{\prime} \subseteq E$ is a spanning subgraph of $G$, and $k\left(G^{\prime}\right)$ denotes the number of (connected) components of $G^{\prime}$. Because (1.4) does not contain any explicit reference to the spins $\left\{\sigma_{i}\right\}$ or summation over spin configurations, it allows one to define the zero-field Potts model partition function with $q$ not necessarily restricted to the positive integers, $\mathbb{N}_{+}$. The zero-field Potts model partition function is equivalent to the Tutte (also called Tutte-Whitney) polynomial $T(G, x, y)$, a function of major importance in mathematical graph theory [6]-[8], [12]-[14] defined by

$$
T(G, x, y)=\sum_{G^{\prime} \subseteq G}(x-1)^{k\left(G^{\prime}\right)-k(G)}(y-1)^{c\left(G^{\prime}\right)},
$$

where $c\left(G^{\prime}\right)=e\left(G^{\prime}\right)+k\left(G^{\prime}\right)-n\left(G^{\prime}\right)$ is the number of independent cycles on $G^{\prime}$. The equivalence is

$$
Z(G, q, v)=(x-1)^{k(G)}(y-1)^{n(G)} T(G, x, y),
$$

where $x=1+(q / v)$, so $q=(x-1)(y-1)$.

In order to treat the Potts model in a magnetic field for non-integral $q$, it is necessary to have a generalization of Eq. (1.4), that is, a formula for the partition function that does not make any explicit reference to the spins or any summation over the spin values, since these spin values are restricted to lie in $\mathbb{N}_{+}$. F. Y. Wu succeeded in constructing such a generalization, which, for an arbitrary graph $G$, expresses $Z(G, q, v, w)$ as a sum of terms from spanning subgraphs $G^{\prime}$ of $G[15$ (see also [2, 5]). Let us label each of the connected components of $G^{\prime}$ as $G_{i}^{\prime}, i=1, \ldots, k\left(G^{\prime}\right)$. Wu's result is $2,5,15$,

$$
Z(G, q, v, w)=\sum_{G^{\prime} \subseteq G} v^{e\left(G^{\prime}\right)} \prod_{i=1}^{k\left(G^{\prime}\right)}\left(q-1+w^{n\left(G_{i}^{\prime}\right)}\right)
$$

Clearly, this formula defines $Z(G, q, v, w)$ in a manner such that $q$ need not be in $\mathbb{N}_{+}$. Eq. (1.7) also shows that $Z(G, q, v, w)$ is a polynomial in the variables $q, v$, and $w$. In the limit $h \rightarrow-\infty$ (i.e., $w \rightarrow 0)$, Eqs. (1.1) and (1.2) show that configurations in which any $\sigma_{i}=1$ make no contribution to $Z$, so that the model reduces to the zero-field case with $q$ replaced by $q-1$ :

$$
Z(G, q, v, 0)=Z(G, q-1, v, 1)
$$

In this paper we present a method for calculating transfer matrices for the $q$-state Potts model partition functions $Z(G, q, v, w)$ in an external magnetic field $H$, for arbitrary $q$ and temperature variable $v$, on cyclic, Möbius and free strip graphs $G$ of the square (sq), triangular (tri), and honeycomb (hc) lattices with width $L_{y}$ vertices and with arbitrarily great length $L_{x}$ vertices. Since this method enables one to calculate $Z(G, q, v, w)$ for arbitrarily great strip lengths, it complements calculations for $L_{x} \times L_{y}$ lattice patches based on enumeration 
of states (e.g., [16]). Using our transfer matrix method, we determine the general structure of this partition function as a sum of powers of the eigenvalues of the transfer matrix, multiplied by certain coefficients that depend only on $q$, not on $v$ or $w$. The result that we find exhibits some interesting differences with the form that has been established for the Potts model partition function on lattice strips in the case of zero external field, and we explain how our more general structure reduces to the zero-field form when the external field vanishes. We shall present explicit results for arbitrary $L_{y}$ given for $T_{Z, \Lambda, L_{y}, d}$ with $d=L_{y}$ and $d=L_{y}-1$, and the determinant $\operatorname{det}\left(T_{Z, \Lambda, L_{y}, d}\right)$. We have calculated the full transfer matrices up to widths $L_{y}=3$ for the square, triangular, and honeycomb lattices and $L_{y}=2$ for the cyclic self-dual strip of the square lattice. Since the total dimensions of these transfer matrices increase very rapidly with strip width, it is not feasible to present many of the explicit results here; instead, we concentrate on general methods and results that hold for arbitrary $L_{y}$. In Ref. [17], besides mentioning briefly our structural results for cyclic lattice strips, we have used the Wu formula (1.7) to derive properties of $Z(G, q, v, w)$, for arbitrary graphs $G$, concerning factorization, monotonicity, and zero-free regions. In Ref. [17] we have also presented a generalization of the Tutte polynomial that corresponds to $Z(G, q, v, w)$ and have formulated and discussed two related weighted graph coloring problems. Some earlier work using transfer matrices for the calculations of the zero-field Potts model partition function for arbitrary $q$ and $v$ on lattice strips of fixed width and arbitrary length is in Refs. [18]-[29]. Transfer matrix and related linear algebraic methods, as well as related generating function methods, have also been used to calculate a particular special case in zero field, namely the chromatic polynomial [13, 14, 30]; references to the literature can be found in reviews such as Refs. [2]-[8].

\section{GENERAL STRUCTURE OF POTTS MODEL PARTITION FUNCTION ON LATTICE STRIPS IN A MAGNETIC FIELD}

\section{A. Basic Method of Analysis and Structure for Cyclic and Möbius Lattice Strips}

In this section we derive the general structural form of the Potts model partition function $Z\left(G_{s}, q, v, w\right)$ in an external magnetic field $H$ on lattice strip graphs $G_{s}$. We label the lattice type as $\Lambda$ and abbreviate the three respective types as $s q, t r i$, and $h c$. Each strip involves a longitudinal repetition of $m$ copies of a particular subgraph. For the square-lattice strips, this is a column of squares. It is convenient to represent the strip of the triangular lattice as obtained from the corresponding strip of the square lattice via the insertion of diagonal edges connecting, say, the upper-left to lower-right vertices in each square. In both of these cases, the length is $L_{x}=m$ vertices. We represent the strip of the honeycomb lattice in the form of bricks oriented horizontally. In this case, since there are two vertices in 1-1 correspondence with each horizontal side of a brick, $L_{x}=2 m$ vertices. Summarizing for all of three lattices, the relation between the number of vertices and the number of repeated copies is

$$
L_{x}= \begin{cases}m & \text { if } \Lambda=s q \text { or } \text { tri or } G_{D} \\ 2 m & \text { if } \Lambda=h c\end{cases}
$$

Here $G_{D}$ is the cyclic self-dual strip of the square lattice, to be discussed further below.

For cyclic strips, the full transfer matrix $T_{Z, \Lambda, L_{y}}$, has a block structure formally specified by

$$
T_{Z, \Lambda, L_{y}}=\bigoplus_{d=0}^{L_{y}} \prod T_{Z, \Lambda, L_{y}, d}
$$

where the product $\prod T_{Z, \Lambda, L_{y}, d}$ means a set of square blocks of the form $\lambda_{Z, \Lambda, L_{y}, d, j}$ times the identity matrix. As indicated, each block is indexed by a non-negative integer $d$, which runs from 0 to $L_{y}$. We shall refer to 
this as the degree of the block. The reason for this terminology is that for $q \geq 5$, this abstract submatrix has a dimension given by a certain polynomial $\tilde{c}^{(d)}$ defined below in Eq. (2.4), which is of degree $d$ in $q$. We shall also refer to the $\lambda_{Z, \Lambda, L_{y}, d}$ in this block as lying in the degree- $d$ subspace of the full space in which the transfer matrix is defined. From Eq. (2.2), it follows that the partition function of the Potts model, in an external magnetic field, on an $L_{x} \times L_{y}$ strip of the lattice $\Lambda$ has the general structural form

$$
\begin{aligned}
Z\left(\Lambda, L_{y} \times L_{x}, c y c ., q, v, w\right) & =\sum_{d=0}^{L_{y}} \tilde{c}^{(d)} \operatorname{Tr}\left[\left(T_{\left.\left.Z, \Lambda, L_{y}, d\right)^{m}\right]}\right.\right. \\
& =\sum_{d=0}^{L_{y}} \tilde{c}^{(d)} \sum_{j=1}^{n_{Z h}\left(\Lambda, L_{y}, d\right)}\left(\lambda_{Z, \Lambda, L_{y}, d, j}\right)^{m}
\end{aligned}
$$

with $m$ given by (2.1). Here the eigenvalues $\lambda_{Z, \Lambda, L_{y}, d, j}$ depend on the lattice type $\Lambda$, the strip width $L_{y}$, and the variables $q, v$, and $w$, but not on the strip length, $L_{x}$. The number of different $\lambda_{Z, \Lambda, L_{y}, d}$ 's in each subspace of degree $d$ is given by $n_{Z h}\left(L_{y}, d\right)$, where we use the symbol $Z h$ to indicate the nonzero field and to distinguish these numbers from the different numbers $n_{Z}\left(L_{y}, d\right)$ for the zero-field case. The coefficients $\tilde{c}^{(d)} \equiv \tilde{c}^{(d)}(q)$ are polynomials of degree $d$ in $q$ defined by:

$$
\tilde{c}^{(d)}=\sum_{j=0}^{d}(-1)^{j}\left(\begin{array}{c}
2 d-j \\
j
\end{array}\right)(q-1)^{d-j}
$$

The first few of these coefficients are $\tilde{c}^{(0)}=1, \tilde{c}^{(1)}=q-2, \tilde{c}^{(2)}=q^{2}-5 q+5, \tilde{c}^{(3)}=q^{3}-8 q^{2}+19 q-13$, and $\tilde{c}^{(4)}=(q-2)\left(q^{3}-9 q^{2}+24 q-17\right)$. For $q \geq 5$, the coefficients $\tilde{c}^{(d)}$ are positive integers and can be interpreted as multiplicities of the corresponding eigenvalues $\lambda_{Z, \Lambda, L_{y}, d}$, or equivalently, as the dimensions of the sub-blocks $T_{Z, \Lambda, L_{y}, d}$ in the transfer matrix $T_{Z, \Lambda, L_{y}}$. Starting from this range of $q$, one can then continue the expression (2.3) to arbitrary $q$. However, for some positive integer $q$ values, the $\tilde{c}^{(d)}$ 's are negative, and hence cannot directly be interpreted as multiplicities of eigenvalues. For example,

$$
\tilde{c}^{(d)}=-1 \quad \text { for } \quad q=2 \quad \text { and } \quad d=2 \bmod 3
$$

and

$$
\tilde{c}^{(d)}=-1 \quad \text { for } \quad q=3 \quad \text { and } \quad d=2 \text { or } 3 \bmod 4
$$

For brevity, we usually suppress the argument in the notation, writing simply $\tilde{c}^{(d)}$ rather than $\tilde{c}^{(d)}(q)$. The coefficients $\tilde{c}^{(d)}$ for the present case of the Potts model in a nonzero magnetic field are related to the corresponding coefficients $c^{(d)}$ 19, 31] for the zero-field Potts model according to the following equation (with arguments indicated explicitly)

$$
\tilde{c}^{(d)}(q)=c^{(d)}(q-1),
$$

where

$$
c^{(d)}=U_{2 d}\left(\frac{\sqrt{q}}{2}\right)=\sum_{j=0}^{d}(-1)^{j}\left(\begin{array}{c}
2 d-j \\
j
\end{array}\right) q^{d-j}
$$

where $U_{n}(x)$ is the Chebyshev polynomial of the second kind. We have also constructed a general formula analogous to Eq. (2.3) for the $Z(G, q, v, w)$ where $G$ is a Möbius strip and a self-dual strip of the square lattice, extending our earlier results for the zero-field case in [31]-[41]. 
To derive Eq. (2.3), let us first consider the subspace of degree $d=0$ in the transfer matrix for a strip with width $L_{y}$ vertices. This matrix is defined with respect to a given basis, and we shall refer to the configurations that comprise this basis as the basis elements. In addition to the basis elements of the transfer matrix for the zero-field case consisting of all the possible non-crossing partitions of $L_{y}$ vertices, there are additional basis elements where certain vertices are in the $q=1$ state. The eigenvalues of the transfer matrix for a free strip are the same as the eigenvalues of the transfer matrix $T_{Z, \Lambda, L_{y}, d=0}$ in this degree $d=0$ subspace for the corresponding cyclic strip, where a set of horizontal edges connecting two adjacent sets of $L_{y}$ vertices do not occur. The dimension of this matrix, denoted as $n_{Z h}\left(L_{y}, 0\right)$, is the binomial transform [34] of a Catalan number,

$$
n_{Z h}\left(L_{y}, 0\right)=\sum_{k=0}^{L_{y}}\left(\begin{array}{c}
L_{y} \\
k
\end{array}\right) C_{k}
$$

where

$$
C_{k}=\frac{1}{k+1}\left(\begin{array}{c}
2 k \\
k
\end{array}\right)
$$

is the Catalan number. (No confusion should result from our use of the same symbol $C_{n}$ to denote the circuit graph with $n$ vertices since the meaning will be clear from context.) Parenthetically, we note that the binomial transform in Eq. (2.9) has been of interest in other combinatorial problems and appears as sequence A007317 in Ref. [35]; some other sequences given here also have appeared in different mathematical contexts and have been similarly catalogued in Ref. [35]. We do not show the dependence on the lattice $\Lambda$ explicitly in $n_{Z h}\left(L_{y}, d\right)$ as it is the same for cyclic strips of the square, triangular and honeycomb lattices as for the zero-field transfer matrices [23, 31]. (Below we shall consider self-dual strips $G_{D}$ of the square lattice, which have different dimensions $n_{Z h}\left(G_{D}, L_{y}, d\right)$; for this case we shall include the $G_{D}$ dependence in the notation.) The degree $d=1$ subspace is given by all of the possible non-crossing partitions with a color assignment, out of $q-1$ states, to one vertex, with possible connections with other vertices, plus the basis elements where certain other vertices are in the $q=1$ state. The multiplicity is given by $\tilde{c}^{(1)}=q-2$. This follows because there are $q-1$ possible ways of making this color assignment, but one of these has to be subtracted, since the effect of all the possible color assignments is equivalent to the choice of no specific color assignment, which has been taken into account in the level 0 subspace. Equivalently, $\tilde{c}^{(1)}(q)=c^{(1)}(q-1)$. In this derivation and subsequent ones we assume that $q$ is an integer $\geq 5$ to begin with, so that the multiplicities are positive-definite; we then analytically continue them downward to apply in the region $0 \leq q<5$ where $\tilde{c}^{(d)}$ can be zero or negative. For the next subspace $d=2$ we consider all of the non-crossing partitions with two-color assignments to two separated vertices (with possible connections with other vertices), plus the basis elements where certain other vertices are in the $q=1$ state. This method is then continued for higher $d$ up to the maximum degree, $d=L_{y}$. The multiplicity $\tilde{c}^{(d)}(q)$ for general $d$ is given by Eq. (2.4).

To illustrate the method further, we list graphically all the possible partitions for the strips with $L_{y}=1$, $L_{y}=2$, and $L_{y}=3$ in Figs. 11-3, where white circles are the original $L_{y}$ vertices, each black circle corresponds to a specific color assignment, and the crosses are the vertices in the $q=1$ state. In the following discussion, we will simply use the names white and black circles and crosses with the meaning understood. We denote the set of partitions $\mathcal{P}_{L_{y}, d}$ for $1 \leq L_{y} \leq 3$ as follows. For simplicity, a single white circle is not given explicitly in the notation for a partition, where contiguous vertex numbers are in the same state (color), vertices with overline are color-assigned, and vertices with underline are in the $q=1$ state. In the following set of partitions $\mathcal{P}_{L_{y}, d}$, individual partitions are separated by a semicolon. For each partition, vertices that are not in the same 
$d=0 \quad \times \quad d=1 \quad \oint$

FIG. 1: Partitions for the $L_{y}=1$ strip.

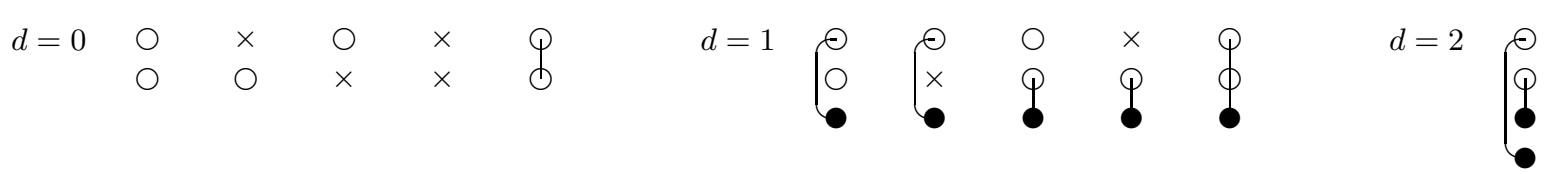

FIG. 2: Partitions for the $L_{y}=2$ strip.

state are separated by a comma.

$$
\begin{gathered}
\mathcal{P}_{1,0}=\{I ; \underline{1}\}, \quad \mathcal{P}_{1,1}=\{\overline{1}\} \\
\mathcal{P}_{2,0}=\{I ; \underline{1} ; \underline{2} ; \underline{12} ; 12\}, \quad \mathcal{P}_{2,1}=\{\overline{1} ; \overline{1}, \underline{2} ; \overline{2} ; \underline{1}, \overline{2} ; \overline{12}\}, \quad \mathcal{P}_{2,2}=\{\overline{1}, \overline{2}\} \\
\mathcal{P}_{3,0}=\{I ; \underline{1} ; \underline{2} ; \underline{3} ; \underline{12} ; \underline{13} ; \underline{23} ; \underline{123} ; 12 ; 12, \underline{3} ; 13 ; 13, \underline{2} ; 23 ; \underline{1}, 23 ; 123\}, \\
\mathcal{P}_{3,1}=\{\overline{3} ; \underline{1}, \overline{3} ; \underline{2}, \overline{3} ; \underline{12}, \overline{3} ; \overline{2} ; \underline{1}, \overline{2} ; \underline{3}, \overline{2} ; \underline{13}, \overline{2} ; \overline{1} ; \overline{1}, \underline{2} ; \overline{1}, \underline{3} ; \overline{1}, \underline{23} ; 12, \overline{3} ; \overline{12} ; \overline{12}, \underline{3} ; \overline{13} ; \overline{13}, \underline{2} ; \overline{23} ; \\
\mathcal{P}_{3,2}=\{\underline{1}, \overline{23} ; \overline{1}, 23 ; \overline{12}\}, \quad\{\overline{3} ; \underline{1}, \overline{2}, \overline{3} ; \overline{1}, \overline{3} ; \overline{1}, \overline{3}, \underline{2} ; \overline{1}, \overline{2} ; \overline{1}, \overline{2}, \underline{3} ; \overline{12}, \overline{3} ; \overline{1}, \overline{23}\}, \quad \mathcal{P}_{3,3}=\{\overline{1}, \overline{2}, \overline{3}\}
\end{gathered}
$$

From Eq. (2.2), it follows that the dimension of the total transfer matrix, i.e., the total number of eigenvalues $\lambda_{Z, \Lambda, L_{y}, d, j}$, counting multiplicities, is

$$
\begin{aligned}
& C_{Z, c y c ., L_{y}}=\operatorname{dim}\left(T_{Z, \Lambda, L_{y}}\right)=\sum_{d=0}^{L_{y}} \operatorname{dim}\left(T_{Z, \Lambda, L_{y}, d}\right)=\sum_{d=0}^{L_{y}} \tilde{c}^{(d)} n_{Z h}\left(L_{y}, d\right)=q^{L_{y}} \\
& \begin{array}{cccccccccccccccc}
d=0 & 0 & \times & 0 & 0 & \times & \times & 0 & \times & \Phi & \Phi & 0 & 0 & 0 & \times & \Phi \\
& 0 & 0 & \times & 0 & \times & 0 & \times & \times & 0 & 0 & 0 & \times & \Phi & \Phi & \phi \\
& 0 & 0 & 0 & \times & 0 & \times & \times & \times & 0 & \times & \ddots & \ddots & 0 & 0 & 0
\end{array}
\end{aligned}
$$

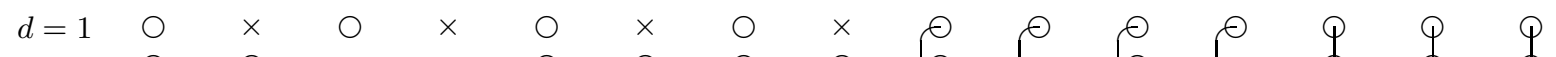

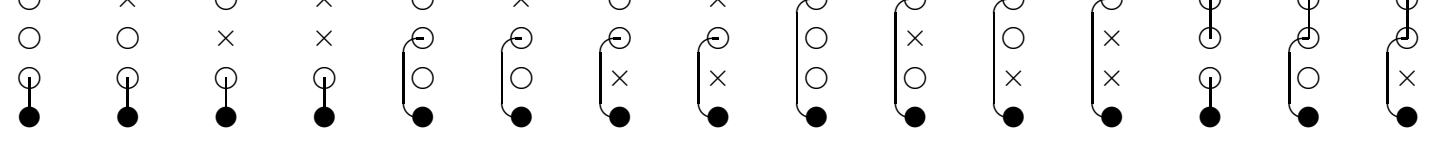

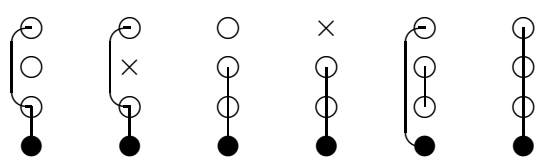

$$
\begin{aligned}
& d=2 \quad \bigcirc \quad \times
\end{aligned}
$$

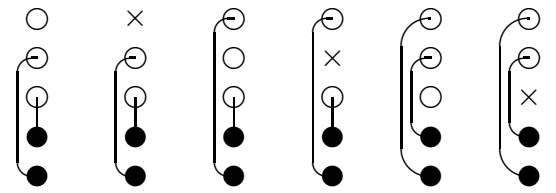

$$
\begin{aligned}
& \int_{0}^{\infty} \int_{0}^{\infty}
\end{aligned}
$$

FIG. 3: Partitions for the $L_{y}=3$ strip. 
which is the sum of coefficients, and is independent of the length $m$ of the strip. We define $N_{Z h, \Lambda, L_{y}}$ as the total number of distinct eigenvalues of $T_{Z, \Lambda, L_{y}}$, i.e. the sum of the dimensions of the submatrices $T_{Z, \Lambda, L_{y}, d}$, modulo the multiplicity $\tilde{c}^{(d)}$ :

$$
N_{Z h, L_{y}}=\sum_{d=0}^{L_{y}} n_{Z h}\left(L_{y}, d\right)
$$

Here we include an $h$ after the $Z$ in the notation $N_{Z h, \Lambda, L_{y}}$ to indicate the presence of a nonzero magnetic field and to avoid confusion with our earlier notation in Ref. 31] for the different (smaller) total number $N_{Z, \Lambda, L_{y}}$ for the zero-field case.

Using these methods, we have determined the $n_{Z h}\left(L_{y}, d\right)$. We find that they are given by the following theorem, whose proof is similar to that for the zero-field case given in [29].

Theorem II.1 The dimension of $T_{Z, \Lambda, L_{y}, d}, n_{Z h}\left(L_{y}, d\right)$, for $H \neq 0$ and $0 \leq d \leq L_{y}$ is determined as follows. One has

$$
\begin{gathered}
n_{Z h}\left(L_{y}, d\right)=0 \text { for } d>L_{y} \\
n_{Z h}\left(L_{y}, L_{y}\right)=1
\end{gathered}
$$

and

$$
n_{Z h}(1,0)=2
$$

All other numbers $n_{Z h}\left(L_{y}, d\right)$ are then determined by the two recursion relations

$$
n_{Z h}\left(L_{y}+1,0\right)=2 n_{Z h}\left(L_{y}, 0\right)+n_{Z h}\left(L_{y}, 1\right)
$$

and

$$
n_{Z h}\left(L_{y}+1, d\right)=n_{Z h}\left(L_{y}, d-1\right)+3 n_{Z h}\left(L_{y}, d\right)+n_{Z h}\left(L_{y}, d+1\right) \quad \text { for } \quad 1 \leq d \leq L_{y}+1
$$

Proof: Since the maximum number of colors to assign is $L_{y}$ for a strip with width $L_{y}$, it follows that $n_{Z h}\left(L_{y}, d\right)=0$ for $d>L_{y}$ and $n_{Z h}\left(L_{y}, L_{y}\right)=1$. It is elementary that $n_{Z h}(1,0)=2$, as shown in Fig. 1 The $d=0$ partitions of a width- $\left(L_{y}+1\right)$ strip can be obtained by either adding a unconnected white circle or a cross to the bottom of the $d=0$ partitions of a width- $L_{y}$ strip, or converting the black circle of the $d=1$ partitions of a width- $L_{y}$ strip into a white circle. This gives Eq. (2.19), which is a special case of the following discussion. For general $0 \leq d \leq L_{y}+1$, the partitions of a width- $\left(L_{y}+1\right)$ strip can be obtained in one of the following four ways: (a) for $1 \leq d \leq L_{y}+1$, adding a pair of connected circles, one black and one white, (but not connected to any other vertex) above the highest black circle of the $d-1$ partitions of a width- $L_{y}$ strip; (b) for $0 \leq d \leq L_{y}$, adding a unconnected white circle or a cross above the highest black circle of the $d$ partitions of a width- $L_{y}$ strip; (c) for $1 \leq d \leq L_{y}$, adding a white circle above the highest black circle of the $d$ partitions of a width- $L_{y}$ strip and connecting these two circles; and (d) for $0 \leq d \leq L_{y}-1$, converting the highest black circle of the $d+1$ partitions of a width- $L_{y}$ strip into a white circle. Now the lowest white circle of the $d$ partitions of a width- $\left(L_{y}+1\right)$ strip can either connect to a black circle with or without ((c) or (a)) other connections to other white circles, or it may not connect to a black circle with or without ((d) or (b)) other connections to other white circles. The case in which the lowest vertex is a cross is included in (b). 
TABLE I: Table of numbers $n_{Z h}\left(L_{y}, d\right)$ and their sums, $N_{Z h, L_{y}}$. Blank entries are zero.

\begin{tabular}{|c|c|c|c|c|c|c|c|c|c|c|c|c|}
\hline \hline$L_{y} \backslash d$ & 0 & 1 & 2 & 3 & 4 & 5 & 6 & 7 & 8 & 9 & 10 & $N_{Z h, L_{y}}$ \\
\hline \hline 1 & 2 & 1 & & & & & & & & & & 3 \\
\hline 2 & 5 & 5 & 1 & & & & & & & & 11 \\
\hline 3 & 15 & 21 & 8 & 1 & & & & & & & & 45 \\
\hline 4 & 51 & 86 & 46 & 11 & 1 & & & & & & & 195 \\
\hline 5 & 188 & 355 & 235 & 80 & 14 & 1 & & & & & 873 \\
\hline 6 & 731 & 1488 & 1140 & 489 & 123 & 17 & 1 & & & & 3989 \\
\hline 7 & 2950 & 6335 & 5397 & 2730 & 875 & 175 & 20 & 1 & & & & 18483 \\
\hline 8 & 12235 & 27352 & 25256 & 14462 & 5530 & 1420 & 236 & 23 & 1 & & 86515 \\
\hline 9 & 51822 & 119547 & 117582 & 74172 & 32472 & 10026 & 2151 & 306 & 26 & 1 & & 408105 \\
\hline 10 & 223191 & 528045 & 546465 & 372570 & 181614 & 64701 & 16785 & 3095 & 385 & 29 & 1 & 1936881 \\
\hline \hline
\end{tabular}

Therefore, (a) to (d) exhaust all the possibilities. The numbers for these four categories are $n_{Z h}\left(L_{y}, d-1\right)$, $2 n_{Z h}\left(L_{y}, d\right), n_{Z h}\left(L_{y}, d\right)$, and $n_{Z h}\left(L_{y}, d+1\right)$, respectively, so we have

$$
\begin{aligned}
n_{Z h}\left(L_{y}+1, d\right) & =n_{Z h}\left(L_{y}, d-1\right)+3 n_{Z h}\left(L_{y}, d\right)+n_{Z h}\left(L_{y}, d+1\right) \quad \text { for } 1 \leq d \leq L_{y}-1 \\
n_{Z h}\left(L_{y}+1, L_{y}\right) & =n_{Z h}\left(L_{y}, L_{y}-1\right)+3 n_{Z h}\left(L_{y}, L_{y}\right) \\
n_{Z h}\left(L_{y}+1, L_{y}+1\right) & =n_{Z h}\left(L_{y}, L_{y}\right)
\end{aligned}
$$

Since $n_{Z h}\left(L_{y}, d\right)=0$ for $d>L_{y}$, these can be combined into Eq. (2.20). The relation for $d=0$, i.e. Eq. (2.19), results from (b) and (d) only. This completes the proof. $\square$ We remark that these results can also be obtained from Eq. (2.14) with $\tilde{c}^{(d)}$ given in Eq. (2.4) by the argument in the proof of Theorem 4 in Ref. [31].

A corollary is that

$$
n_{Z h}\left(L_{y}, L_{y}-1\right)=3 L_{y}-1
$$

In Table \ we list the first few numbers $n_{Z h}\left(L_{y}, d\right)$ and the total sums $N_{Z h, L_{y}}$. In particular, the numbers $n_{Z h}\left(L_{y}, 0\right)$ have been given in Eq. (2.9), and the numbers $n_{Z h}\left(L_{y}, 1\right)$ are the binomial transforms of the first differences of the Catalan numbers.

Two corollaries of Theorem II.1 are the following. First,

$$
N_{Z h, L_{y}+1}=5 N_{Z h, L_{y}}-2 n_{Z h}\left(L_{y}, 0\right)
$$

Second, $N_{Z h, L_{y}}$ can be expressed as

$$
N_{Z h, L_{y}}=\sum_{j=0}^{L_{y}}\left(\begin{array}{c}
L_{y} \\
j
\end{array}\right)\left(\begin{array}{c}
2 j \\
j
\end{array}\right)
$$

The construction of the transfer matrix for each level (i.e., degree) $d$ can be carried out by methods similar to those for the zero-field transfer matrix [29]. Using the basis elements described above (e.g. Eqs. (2.11)-(2.13) for $\left.1 \leq L_{y} \leq 3\right)$, we define $J_{L_{y}, d, i, i+1}$ as the join operator between vertices $i$ and $i+1$, i.e.

$$
J_{L_{y}, d, i, i+1} \mathbf{v}_{\mathcal{P}}=\mathbf{v}_{\mathcal{P} i(i+1)},
$$


where both of these vertices $i$ and $i+1$ are in states different from the $q=1$ state and have not already been assigned different colors. $\mathcal{P} i(i+1)$ denotes the partition with $d$ color assignments obtained from $\mathcal{P}$ by connecting vertices $i$ and $i+1$ (regardless of whether they were already connected or not). For each subspace $d$, we also define $D_{L_{y}, d, i}$ as the detach operator on vertex $i$ such that

$$
D_{L_{y}, d, i} \mathbf{v}_{\mathcal{P}}=\left\{\begin{array}{ll}
\mathbf{v}_{\mathcal{P} \backslash i}+\mathbf{v}_{\mathcal{P} \backslash \underline{i}} & \text { if } i \text { is a cross or it is connected to other vertices } \\
(q-1)\left(\mathbf{v}_{\mathcal{P} i}+\mathbf{v}_{\mathcal{P} \underline{i}}\right) & \text { if } i \text { is a white circle without connection }
\end{array},\right.
$$

where the vertex $i$ should not have been assigned color. $\mathcal{P} \backslash i$ is the partition obtained from $\mathcal{P}$ by making $i$ a white circle without connection, and similarly, $\mathcal{P} \backslash \underline{i}$ is the partition obtained from $\mathcal{P}$ by making $i$ a cross. $\mathcal{P} i$ is the same as $\mathcal{P}$ if $i$ has originally no connection, and $\mathcal{P} \underline{i}$ is the partition obtained from $\mathcal{P}$ by converting $i$ into a cross. Since the application of these operators does not increase the number of colors assigned, i.e. $q-1$, the full transfer matrix in Eq. (2.2) has a triangular block (submatrix) form, and the block corresponding to a $d$-color assignment has a diagonal block form with $\tilde{c}^{(d)}$ blocks. The transfer matrix $T_{Z, \Lambda, L_{y}, d}$ for each $d$ is the product of the transverse and longitudinal parts, $H_{Z, \Lambda, L_{y}, d}$ and $V_{Z, \Lambda, L_{y}, d}$, which can be expressed as

$$
\begin{aligned}
H_{Z, s q, L_{y}, d} & =H_{Z, t r i, L_{y}, d}=K \prod_{i=1}^{L_{y}-1}\left(I+v J_{L_{y}, d, i, i+1}\right) \\
H_{Z, h c, L_{y}, d, 1} & =K \prod_{i=1}^{\left[L_{y} / 2\right]}\left(I+v J_{L_{y}, d, 2 i-1,2 i}\right), \quad H_{Z, h c, L_{y}, d, 2}=K \prod_{i=1}^{\left[\left(L_{y}-1\right) / 2\right]}\left(I+v J_{L_{y}, d, 2 i, 2 i+1}\right) \\
V_{Z, s q, L_{y}, d} & =V_{Z, h c, L_{y}, d}=\prod_{i=1}^{L_{y}}\left(v I+D_{L_{y}, d, i}\right) \\
V_{Z, t r i, L_{y}, d} & =\prod_{i=1}^{L_{y}-1}\left[\left(v I+D_{L_{y}, d, i}\right)\left(I+v J_{L_{y}, d, i, i+1}\right)\right]\left(v I+D_{L_{y}, d, L_{y}}\right)
\end{aligned}
$$

where $[\nu]$ denotes the integral part of $\nu$. Here $K$ is the diagonal matrix with diagonal element $w^{\ell}$, where $\ell$ is the number of vertices in the $q=1$ state for the corresponding basis element. We have

$$
\begin{aligned}
& T_{Z, s q, L_{y}, d}=V_{Z, s q, L_{y}, d} H_{Z, s q, L_{y}, d}, \quad T_{Z, t r i, L_{y}, d}=V_{Z, t r i, L_{y}, d} H_{Z, t r i, L_{y}, d} \\
& T_{Z, h c, L_{y}, d}=\left(V_{Z, h c, L_{y}, d} H_{Z, h c, L_{y}, d, 2}\right)\left(V_{Z, h c, L_{y}, d} H_{Z, h c, L_{y}, d, 1}\right) \equiv T_{Z, h c, L_{y}, d, 2} T_{Z, h c, L_{y}, d, 1}
\end{aligned}
$$

We explain various details for both cyclic and Möbius strips. Consider two adjacent sets of $L_{y}$ vertices and denote these as $i_{1}, i_{2}, \ldots i_{L_{y}}$ and $j_{1}, j_{2}, \ldots j_{L_{y}}$. For cyclic strips of the square lattice, the horizontal edges connecting these vertices are $\left(i_{1}, j_{1}\right),\left(i_{2}, j_{2}\right), \ldots,\left(i_{L_{y}}, j_{L_{y}}\right)$. For Möbius strips, one set of horizontal edges becomes $\left(i_{1}, j_{L_{y}}\right),\left(i_{2}, j_{L_{y}-1}\right), \ldots,\left(i_{L_{y}}, j_{1}\right)$. This corresponds to exchanging the pair of basis elements that switch to each other when the vertices reverse in order, i.e., the set of basis elements that do not have selfreflection symmetry with respect to the center of the $L_{y}$ vertices. For example, among the partitions for the $L_{y}=2$ strip in Fig. 2, the second partition $\underline{1}$ and the third partition $\underline{2}$ in $\mathcal{P}_{2,0}$ must be exchanged under this reflection. Similarly, the pairs of partitions in $\mathcal{P}_{2,1}$ are (i) the first partition $\overline{1}$ and the third partition $\overline{2}$, (ii) the second partition $\overline{1}, \underline{2}$ and the forth partition $\underline{1}, \overline{2}$. For this specific set of edges of Möbius strips, the pairs of columns of $V_{Z, \Lambda, L_{y}, d}$ that correspond to these pairs of partitions should be exchanged, and these matrices will be denoted as $\hat{V}_{Z, \Lambda, L_{y}, d}$. Equivalently, the same pairs of columns of $T_{Z, \Lambda, L_{y}, d}$ should be exchanged, and these matrices will be denoted as $\hat{T}_{Z, \Lambda, L_{y}, d}=\hat{V}_{Z, \Lambda, L_{y}, d} H_{Z, \Lambda, L_{y}, d}$ for $\Lambda=s q$, tri. There are two kinds of Möbius strips for the honeycomb lattice. When $L_{y}$ is even, the number of vertices in the horizontal direction is even 
as for the cyclic strips, i.e., $L_{x}=2 \mathrm{~m}$. When $L_{y}$ is odd, the number of vertices in the horizontal direction is odd, $L_{x}=2 m-1$. Therefore, for the honeycomb lattice, we use the definition

$$
\begin{aligned}
& \hat{T}_{Z, h c, L_{y}, d}=\hat{V}_{Z, h c, L_{y}, d} H_{Z, h c, L_{y}, d, 1} \quad \text { for odd } L_{y} \\
& \hat{T}_{Z, h c, L_{y}, d}=\hat{V}_{Z, h c, L_{y}, d} H_{Z, h c, L_{y}, d, 2} V_{Z, h c, L_{y}, d} H_{Z, h c, L_{y}, d, 1} \quad \text { for even } L_{y}
\end{aligned}
$$

As was discussed in 23] for the crossing-subgraph strips, the square of each eigenvalue of $\hat{T}_{Z, h c, L_{y}, d}$ for odd $L_{y}$ is an eigenvalue of the corresponding $T_{Z, h c, L_{y}, d}$.

We now apply these general methods to determine the structure of the Potts model partition function in a magnetic field on a lattice strip with Möbius boundary conditions. In the case of zero external magnetic field, we previously determined the changes of coefficients $c^{(d)}$ when the longitudinal boundary condition is changed from cyclic to Möbius [29, 31]. Here we have the same changes of coefficients $\tilde{c}^{(d)}$ for the square, triangular and honeycomb lattices, as follows:

$$
\begin{gathered}
\tilde{c}^{(0)} \rightarrow \tilde{c}^{(0)} \\
\tilde{c}^{(2 k)} \rightarrow-\tilde{c}^{(k-1)}, \quad 1 \leq k \leq\left[\frac{L_{y}}{2}\right]
\end{gathered}
$$

and

$$
\tilde{c}^{(2 k+1)} \rightarrow \tilde{c}^{(k+1)}, \quad 0 \leq k \leq\left[\frac{L_{y}-1}{2}\right]
$$

We thus find the following general structure for the Potts model partition function for Möbius strips:

$$
\begin{aligned}
Z\left(\Lambda, L_{y} \times L_{x}, M b, q, v, w\right)= & \tilde{c}^{(0)} \operatorname{Tr}\left[\left(T_{Z, \Lambda, L_{y}, 0}\right)^{m-1} \hat{T}_{Z, \Lambda, L_{y}, 0}\right] \\
& +\sum_{d=0}^{\left[\left(L_{y}-1\right) / 2\right]} \tilde{c}^{(d+1)} \operatorname{Tr}\left[\left(T_{Z, \Lambda, L_{y}, 2 d+1}\right)^{m-1} \hat{T}_{Z, \Lambda, L_{y}, 2 d+1}\right] \\
& -\sum_{d=1}^{\left[L_{y} / 2\right]} \tilde{c}^{(d-1)} \operatorname{Tr}\left[\left(T_{Z, \Lambda, L_{y}, 2 d}\right)^{m-1} \hat{T}_{Z, \Lambda, L_{y}, 2 d}\right]
\end{aligned}
$$

For the square lattice or the honeycomb lattice with $L_{y}$ even, the eigenvalues of $\hat{T}_{Z, \Lambda, L_{y}, d}$ are the same as those of $T_{Z, \Lambda, L_{y}, d}$ except for possible changes of signs. The number of eigenvalues with sign changes is equal to the number of column-exchanges from $T_{Z, \Lambda, L_{y}, d}$ to $\hat{T}_{Z, \Lambda, L_{y}, d}$. Denote the number of eigenvalues that are the same for $T_{Z, s q, L_{y}, d}$ and $\hat{T}_{Z, s q, L_{y}, d}$ as $n_{Z h}\left(s q, L_{y}, d,+\right)$, and the number of eigenvalues with different signs as $n_{Z h}\left(s q, L_{y}, d,-\right)$. It is clear that

$$
n_{Z h}\left(L_{y}, d\right)=n_{Z h}\left(s q, L_{y}, d,+\right)+n_{Z h}\left(s q, L_{y}, d,-\right)
$$

Define

$$
\Delta n_{Z h}\left(s q, L_{y}, d\right) \equiv n_{Z h}\left(s q, L_{y}, d,+\right)-n_{Z h}\left(s q, L_{y}, d,-\right)
$$

which gives the number of partitions that have self-reflection symmetry. For example, among the partitions for the $L_{y}=2$ strip in Fig. 22 the partitions $I, \underline{12}$ and 12 in $\mathcal{P}_{2,0}$, the fifth partition $\overline{12}$ in $\mathcal{P}_{2,1}$, and the partition $\overline{1}, \overline{2}$ in $\mathcal{P}_{2,2}$ have self-reflection symmetry. Among the partitions for the $L_{y}=3$ strip in Fig. 3 those 
with self-reflection symmetry include (i) the first partition $I$, the third partition $\underline{2}$, the sixth partition $\underline{13}$, the eighth partition $\underline{123}$, the eleven partition 13, the twelve partition 13, $\underline{2}$ and the fifteenth partition 123 in $\mathcal{P}_{3,0}$, (ii) the fifth partition $\overline{2}$, the eighth partition $\overline{2}, \underline{13}$, the sixteenth partition $\overline{13}$, the seventeenth partition $\overline{13}, \underline{2}$, and the twenty first partition $\overline{123}$ in $\mathcal{P}_{3,1}$, (iii) the third partition $\overline{1}, \overline{3}$ and the fourth partition $\overline{1}, \overline{3}, \underline{2}$ in $\mathcal{P}_{3,2}$, and (iv) the partition in $\mathcal{P}_{3,3}$. We list $\Delta n_{Z h}\left(s q, L_{y}, d\right)$ for $1 \leq L_{y} \leq 10$ in Table II The relations between $\Delta n_{Z h}\left(s q, L_{y}, d\right)$ are

$$
\begin{aligned}
\Delta n_{Z h}(s q, 2 n, 0)= & 2 \Delta n_{Z h}(s q, 2 n-1,0)-\Delta n_{Z h}(s q, 2 n-2,0) \quad \text { for } 1 \leq n \\
\Delta n_{Z h}(s q, 2 n+1,0)= & 2 \Delta n_{Z h}(s q, 2 n, 0)+\Delta n_{Z h}(s q, 2 n, 1) \quad \text { for } 0 \leq n \\
\Delta n_{Z h}(s q, 2 n, 2 m-1)= & \Delta n_{Z h}(s q, 2 n, 2 m) \\
= & \Delta n_{Z h}(s q, 2 n-1,2 m-1)+\Delta n_{Z h}(s q, 2 n-1,2 m) \\
& -\Delta n_{Z h}(s q, 2 n-2,2 m-1) \quad \text { for } 1 \leq m \leq n \\
\Delta n_{Z h}(s q, 2 n+1, m)= & \Delta n_{Z h}(s q, 2 n, m-1)+\Delta n_{Z h}(s q, 2 n, m) \\
& +\Delta n_{Z h}(s q, 2 n, m+1) \quad \text { for } 1 \leq m \leq 2 n+1,
\end{aligned}
$$

where the formal quantity $\Delta n_{Z h}(0, d)=\delta_{d, 0}$ is assumed. Closed-form expressions are given by

$$
\begin{aligned}
\Delta n_{Z h}(s q, 2 n, 2 m) & =\sum_{j=0}^{n}\left(\begin{array}{l}
n \\
j
\end{array}\right)\left(\begin{array}{c}
2 j \\
j+m
\end{array}\right) \quad \text { for } 0 \leq m \leq n \\
\Delta n_{Z h}(s q, 2 n+1, m) & = \begin{cases}\sum_{j=0}^{n}\left(\begin{array}{c}
n \\
j
\end{array}\right)\left(\begin{array}{c}
2 j \\
j+m / 2
\end{array}\right) \frac{6 j+m+4}{2 j+m+2} & \text { for even } 0 \leq m \leq 2 n \\
\sum_{j=0}^{n}\left(\begin{array}{c}
n \\
j
\end{array}\right)\left(\begin{array}{c}
2 j \\
j+(m-1) / 2
\end{array}\right) \frac{6 j-m+3}{2 j+m+1} & \text { for odd } 1 \leq m \leq 2 n+1\end{cases}
\end{aligned}
$$

The total number of these partitions for each $L_{y}$, denoted as $\Delta N_{Z h, L_{y}}$, is

$$
\Delta N_{Z h, L_{y}}=\sum_{d=0}^{L_{y}} \Delta n_{Z h}\left(s q, L_{y}, d\right)= \begin{cases}5^{L_{y} / 2} & \text { for even } L_{y} \\ 3 \times 5^{\left(L_{y}-1\right) / 2} & \text { for odd } L_{y}\end{cases}
$$

\section{B. Structure of $Z(G, q, v, w)$ for Free Lattice Strips}

Using the methods discussed above, we find that the Potts model partition function in a magnetic field, on a lattice strips with free boundary conditions, has the form

$$
\begin{aligned}
& Z\left(\Lambda, L_{y} \times L_{x}, \text { free }, q, v, w\right)=u_{L_{y}}^{\mathrm{T}} H_{Z, \Lambda, L_{y}, 0}\left(T_{Z, \Lambda, L_{y}, 0}\right)^{L_{x}-1} s_{L_{y}} \quad \text { for } \Lambda=s q, \text { tri } \\
& Z\left(h c, L_{y} \times L_{x}, \text { free }, q, v, w\right)=u_{L_{y}}^{\mathrm{T}} H_{Z, h c, L_{y}, 0,1}\left(T_{Z, h c, L_{y}, 0}\right)^{\left[\left(L_{x}-1\right) / 2\right]}\left(T_{Z, h c, L_{y}, 0,2}\right)^{\delta} s_{L_{y}},
\end{aligned}
$$

where $\delta$ is defined by

$$
\delta= \begin{cases}1 & \text { for even } L_{y} \\ 0 & \text { for odd } L_{y}\end{cases}
$$


TABLE II: Table of $\Delta n_{Z h}\left(s q, L_{y}, d\right)$ for strips of the square lattice. Blank entries are zero. The last entry for each value of $L_{y}$ is the total number of partitions with self-reflection symmetry.

\begin{tabular}{|c|c|c|c|c|c|c|c|c|c|c|c|}
\hline$L_{y} \backslash d$ & 0 & 1 & 2 & 3 & 4 & 5 & \begin{tabular}{|l|}
6 \\
\end{tabular} & 7 & \begin{tabular}{|l|l}
8 \\
\end{tabular} & \begin{tabular}{l|l|}
9 & 11 \\
\end{tabular} & \begin{tabular}{l|l}
0 & $\Delta N_{Z h, L_{y}}$ \\
\end{tabular} \\
\hline 1 & 2 & 1 & & & & & & & & & 3 \\
\hline 2 & 3 & 1 & 1 & & & & & & & & 5 \\
\hline 3 & 7 & 5 & 2 & 1 & & & & & & & 15 \\
\hline 4 & 11 & 6 & 6 & 1 & 1 & & & & & & 25 \\
\hline 5 & 28 & 23 & 13 & 8 & 2 & 1 & & & & & 75 \\
\hline 6 & 45 & 30 & 30 & 9 & 9 & 1 & 1 & & & & 125 \\
\hline 7 & 120 & 105 & 69 & 48 & 19 & 11 & $2 \mid$ & 1 & & & 375 \\
\hline 8 & 195 & 144 & 144 & 58 & 58 & 12 & 12 & 1 & 1 & & 625 \\
\hline 9 & 534 & 483 & 346 & 260 & 128 & 82 & 25 & 14 & \begin{tabular}{|l|l|}
2 \\
\end{tabular} & 1 & 1875 \\
\hline 10 & 873 & 685 & 685 & 330 & 330 & 95 & 95 & 15 & 15 & \begin{tabular}{|l|l}
1 & 1 \\
\end{tabular} & 3125 \\
\hline
\end{tabular}

The element of the vector $u_{L_{y}}$ for the partition $\mathcal{P}$ is given by $(q-1)^{|\mathcal{P}|}$, where $|\mathcal{P}|$ is the number of components for $\mathcal{P}$ that are not in the $q=1$ state. The element of the vector $s_{L_{y}}$ for the partition $\mathcal{P}$ is equal to unity if there are no connections in $\mathcal{P}$, where certain vertices can be in the $q=1$ state. As an example, $u_{2}^{\mathrm{T}}=$ $\left((q-1)^{2}, q-1, q-1,1, q-1\right)$ and $s_{2}^{\mathrm{T}}=(1,1,1,1,0)$ for $L_{y}=2$. For a strip with free boundary conditions, only the $d=0$ transfer matrix for the strip with cyclic boundary conditions is needed. For the square lattice or the honeycomb lattice with $L_{y}$ even, the size of transfer matrix can be reduced to $n_{Z h}\left(s q, L_{y}, 0,+\right)$ due to reflection symmetry as for the zero-field case [27]. We list $n_{Z h}\left(s q, L_{y}, d,+\right)$ and $n_{Z h}\left(s q, L_{y}, d,-\right)$ for $1 \leq L_{y} \leq 10$ in Table III] From Eqs. (2.34) and (2.35), these are given by

$$
\begin{aligned}
& n_{Z h}\left(s q, L_{y}, d,+\right)=\frac{1}{2}\left[n_{Z h}\left(L_{y}, d\right)+\Delta n_{Z h}\left(s q, L_{y}, d\right)\right] \\
& n_{Z h}\left(s q, L_{y}, d,-\right)=\frac{1}{2}\left[n_{Z h}\left(L_{y}, d\right)-\Delta n_{Z h}\left(s q, L_{y}, d\right)\right]
\end{aligned}
$$

As we did for the zero-field case in Ref. [31], we define, for the case of nonzero field, the numbers of $\lambda_{Z, s q, L_{y}, j}$ for the Möbius strips of the square lattice with coefficients $\pm c^{(d)}$ as $n_{Z h, M b}\left(s q, L_{y}, d, \pm\right)$. We list $n_{Z h, M b}\left(s q, L_{y}, d,+\right)$ and $n_{Z h, M b}\left(s q, L_{y}, d,-\right)$ for $1 \leq L_{y} \leq 10$ in Table IV] With the Eqs. (2.30) to (2.32), the relations between $n_{Z h}\left(s q, L_{y}, d, \pm\right)$ and $n_{Z h, M b}\left(s q, L_{y}, d, \pm\right)$ are

$$
\begin{aligned}
n_{Z h, M b}\left(s q, L_{y}, 0, \pm\right)= & n_{Z h}\left(s q, L_{y}, 0, \pm\right)+n_{Z h}\left(s q, L_{y}, 2, \mp\right) \\
n_{Z h, M b}\left(s q, L_{y}, k, \pm\right)= & n_{Z h}\left(s q, L_{y}, 2 k-1, \pm\right)+n_{Z h}\left(s q, L_{y}, 2 k+2, \mp\right) \\
& \text { for } 1 \leq k \leq\left[\frac{L_{y}+1}{2}\right]
\end{aligned}
$$

The differences for each $d$ are defined as

$$
\Delta n_{Z h, M b}\left(s q, L_{y}, d\right)=n_{Z h, M b}\left(s q, L_{y}, d,+\right)-n_{Z h, M b}\left(s q, L_{y}, d,-\right)
$$

For the Möbius strip of the square lattice or the honeycomb lattice with $L_{y}$ even, the sign changes of the eigenvalues of $\hat{T}_{Z, \Lambda, L_{y}, d}$ can be considered as the sign changes of the coefficients. For these cases, the sum of 
TABLE III: Table of numbers $n_{Z h}\left(s q, L_{y}, d, \pm\right)$ for strips of the square lattice. For each $L_{y}$ value, the entries in the first and second lines are $n_{Z h}\left(s q, L_{y}, d,+\right)$ and $n_{Z h}\left(s q, L_{y}, d,-\right)$, respectively. Blank entries are zero. The last entry for each value of $L_{y}$ is the total $N_{Z h, L_{y}}$.

\begin{tabular}{|c|c|c|c|c|c|c|c|c|c|c|c|c|}
\hline \hline $\begin{array}{r}(d,+) \\
L_{y} \backslash(d,-)\end{array}$ & $0,+$ & $1,+$ & $2,+$ & $3,+$ & $4,+$ & $5,+$ & $6,+$ & $7,+$ & $8,+$ & $9,+$ & $10,+$ & $N_{Z h, L_{y}}$ \\
$1,-$ & $2,-$ & $3,-$ & $4,-$ & $5,-$ & $6,-$ & $7,-$ & $8,-$ & $9,-$ & $10,-$ & \\
\hline \hline 1 & 2 & 1 & & & & & & & & & & 3 \\
& & & & & & & & & & & & \\
\hline 2 & 4 & 3 & 1 & & & & & & & & & 11 \\
& 1 & 2 & & & & & & & & & & \\
\hline 3 & 11 & 13 & 5 & 1 & & & & & & & & 45 \\
& 4 & 8 & 3 & & & & & & & & & \\
\hline 4 & 31 & 46 & 26 & 6 & 1 & & & & & & & 195 \\
& 20 & 40 & 20 & 5 & & & & & & & & \\
\hline 5 & 108 & 189 & 124 & 44 & 8 & 1 & & & & & & 873 \\
& 80 & 166 & 111 & 36 & 6 & & & & & & & \\
\hline 6 & 388 & 759 & 585 & 249 & 66 & 9 & 1 & & & & & 3989 \\
& 343 & 729 & 555 & 240 & 57 & 8 & & & & & & \\
\hline 7 & 1535 & 3220 & 2733 & 1389 & 447 & 93 & 11 & 1 & & & & 18483 \\
& 1415 & 3115 & 2664 & 1341 & 428 & 82 & 9 & & & & & \\
\hline 8 & 6215 & 13748 & 12700 & 7260 & 2794 & 716 & 124 & 12 & 1 & & & 86515 \\
& 6020 & 13604 & 12556 & 7202 & 2736 & 704 & 112 & 11 & & & & \\
\hline 9 & 26178 & 60015 & 58964 & 37216 & 16300 & 5054 & 1088 & 160 & 14 & 1 & & 408105 \\
& 25644 & 59532 & 58618 & 36956 & 16172 & 4972 & 1063 & 146 & 12 & & & \\
\hline 10 & 112032 & 264365 & 273575 & 186450 & 90972 & 32398 & 8440 & 1555 & 200 & 15 & 1 & 1936881 \\
& 111159 & 263680 & 272890 & 186120 & 90642 & 32303 & 8345 & 1540 & 185 & 14 & & \\
\hline \hline
\end{tabular}

coefficients is given by:

$$
C_{Z, s q, L_{y}, M b} \equiv \sum_{j=1}^{N_{Z h, L_{y}, \lambda}} c_{Z, L_{y}, M b, j}=\sum_{d=0}^{d_{\max }} \Delta n_{Z h, M b}\left(s q, L_{y}, d\right) \tilde{c}^{(d)}= \begin{cases}q^{L_{y} / 2} & \text { for even } L_{y} \\ q^{\left(L_{y}+1\right) / 2} & \text { for odd } L_{y}\end{cases}
$$

where

$$
d_{\max }= \begin{cases}\frac{L_{y}}{2} & \text { for even } L_{y} \\ \frac{\left(L_{y}+1\right)}{2} & \text { for odd } L_{y}\end{cases}
$$

That is,

$$
\Delta n_{Z h, M b}\left(s q, 2 L_{y}-1, d\right)=\Delta n_{Z h, M b}\left(s q, 2 L_{y}, d\right)=n_{Z h}\left(L_{y}, d\right) \quad \text { for } 0 \leq d \leq L_{y}
$$

In previous work we have given zero-field results for the determinants for various strip graphs $G_{s}$ (e.g., 24])

$$
\operatorname{det} T_{Z}\left(G_{s}\right)=\prod_{j=1}^{N_{Z, G_{s}, \lambda}}\left(\lambda_{Z, G_{s}, j}\right)^{c_{Z, G_{s}, j}}
$$

where $c_{Z, G_{s}, j}$ is the multiplicity of $\lambda_{Z, G_{s}, j}$. In the present context, these can be written, for cyclic strips, as

$$
\operatorname{det}\left(T_{Z, \Lambda, L_{y}}\right)=\prod_{d=0}^{L_{y}}\left[\operatorname{det}\left(T_{Z, \Lambda, L_{y}, d}\right)\right]^{\tilde{c}^{(d)}}
$$


TABLE IV: Table of numbers $n_{Z h, M b}\left(s q, L_{y}, d, \pm\right)$ for Möbius strips of the square lattice. For each $L_{y}$ value, the entries in the first and second lines are $n_{Z h, M b}\left(s q, L_{y}, d,+\right)$ and $n_{Z h, M b}\left(s q, L_{y}, d,-\right)$, respectively. Blank entries are zero. The last entry for each value of $L_{y}$ is the total $N_{Z h, L_{y}}$.

\begin{tabular}{|c|c|c|c|c|c|c|c|}
\hline \hline $\begin{array}{r}(d,+) \\
L_{y} \backslash(d,-)\end{array}$ & $0,+$ & $1,+$ & $2,+$ & $3,+$ & $4,+$ & $5,+$ & $N_{Z h, L_{y}}$ \\
$1,-$ & $2,-$ & $3,-$ & $4,-$ & $5,-$ & \\
\hline \hline 1 & 2 & 1 & & & & & 3 \\
& & & & & & & \\
\hline 2 & 4 & 3 & & & & & 11 \\
& 2 & 2 & & & & & \\
\hline 3 & 14 & 13 & 1 & & & & 45 \\
& 9 & 8 & & & & & \\
\hline 4 & 51 & 46 & 6 & & & & 195 \\
& 46 & 41 & 5 & & & & \\
\hline 5 & 219 & 195 & 44 & 1 & & & 873 \\
& 204 & 174 & 36 & & & & \\
\hline 6 & 943 & 816 & 249 & 9 & & & 3989 \\
& 928 & 795 & 241 & 8 & & & \\
\hline 7 & 4199 & 3648 & 1398 & 93 & 1 & & 18483 \\
& 4148 & 3562 & 1352 & 82 & & & \\
\hline 8 & 18771 & 16484 & 7372 & 716 & 12 & & 86515 \\
& 18720 & 16398 & 7326 & 705 & 11 & & \\
\hline 9 & 84796 & 76187 & 38279 & 5066 & 160 & 1 & 408105 \\
& 84608 & 75832 & 38044 & 4986 & 146 & & \\
\hline 10 & 384922 & 355007 & 194795 & 32583 & 1555 & 15 & 1936881 \\
& 384734 & 354652 & 194560 & 32503 & 1541 & 14 & \\
\hline \hline
\end{tabular}

and we shall extend these results to arbitrary width with a general, nonzero magnetic field below.

\section{PROPERTIES OF TRANSFER MATRICES AT SPECIAL VALUES OF PARAMETERS}

In this section we derive some properties of the transfer matrices $T_{Z, \Lambda, L_{y}, d}$ at special values of $q$, $v$, and $w$. Some related factorizations were given in [17].

$$
\text { A. } v=0
$$

From (1.7) it follows that for any graph $G$, the Potts model partition function $Z(G, q, v, w)$ at $v=0$ satisfies

$$
Z(G, q, 0, w)=(q-1+w)^{n(G)}
$$

Since this holds for arbitrary values of $q$, in the context of the lattice strips considered here, it implies

$$
\left(T_{Z, \Lambda, L_{y}, d}\right)_{v=0}=0 \quad \text { for } \quad 1 \leq d \leq L_{y}
$$


i.e. these are zero matrices. Secondly, restricting to cyclic strips for simplicity, and using the basic results $n=L_{y} L_{x}=L_{y} m$ for $\Lambda=s q$, tri and $n=2 L_{y} m$ for $\Lambda=h c$, Eq. (3.1) implies that

$$
\operatorname{Tr}\left[\left(T_{Z, \Lambda, c y c ., L_{y}}\right)^{m}\right]_{v=0}= \begin{cases}(q-1+w)^{L_{y} m} & \text { for } \Lambda=s q, \operatorname{tri} \\ (q-1+w)^{2 L_{y} m} & \text { for } \Lambda=h c\end{cases}
$$

With our explicit calculations, we find that all of the eigenvalues of the matrix $T_{Z, \Lambda, L_{y}, d=0}$ for $v=0$ vanish except for one, which is equal to $(q-1+w)^{L_{y}}$ if $\Lambda=s q$, tri and $(q-1+w)^{2 L_{y}}$ if $\Lambda=h c$. As will be seen, this is reflected in the property that $\operatorname{det}\left(T_{Z, \Lambda, L_{y}, d}\right)$ has a nonzero power of $v$ as a factor for $L_{y} \geq 2$ for all of the lattice strips considered here. The restriction $L_{y} \geq 2$ is made because the strips of the triangular and honeycomb lattice are only well-defined without degenerating for $L_{y} \geq 2$. In the case of the square lattice, for the case $L_{y}=1$, the transfer matrices $T_{Z, s q, 1,0}$ also has one eigenvalue $q-1+w$ and the other one equal to zero at $v=0$, and $T_{Z, s q, 1,1}$ is the scalar $v$.

\section{B. $v=-1$}

The special case $v=-1$ defines two new types of weighted graph coloring problems, as we have discussed in [17]. We recall that the chromatic polynomial $P(G, q)$ counts the number of ways of assigning $q$ colors to the vertices of a graph $G$ such that no adjacent vertices have the same color. This "proper $q$-coloring" of the vertices of $G$ is equivalent to $Z$ for the zero-temperature, zero-field Potts antiferromagnet, $v=-1$ : $P(G, q)=Z(G, q,-1)$. Here we have a generalization of this to a weighted proper $q$-coloring of the vertices of $G$, as described by the polynomial [17]

$$
\operatorname{Ph}(G, q, w)=Z(G, q,-1, w)
$$

For $H<0$, i.e., $0 \leq w<1$, this is a weighted graph coloring problem in which one carries out a proper $q$-coloring of the vertices of $G$ but with a penalty factor of $w$ for each vertex assigned the color 1 . For $H>0$, this is a second type of weighted graph coloring problem, namely a proper vertex $q$ coloring with a weighting that favors one color. Since this favoring of one color conflicts with the strict constraint that no two adjacent vertices have the same color, the range $w>1$ involves competing interactions and frustration. In the limit $w \rightarrow \infty$, it is impossible to satisfy the proper coloring constraint, and this is embodied in the analytic result that for large positive $w, Z(G, q, v, w) \sim(v+1)^{e(G)} w^{n(G)}$, which vanishes as $v \rightarrow-1$. In this $v=-1$ special case, there are reductions in the ranks of the transfer matrices $T_{Z, \Lambda, L_{y}, d}$, i.e., some of the eigenvalues vanish. This yields a new set of dimensions of matrix blocks, $n_{P h}\left(L_{y}, d\right) \leq n_{Z h}\left(L_{y}, d\right)$. This is a strict inequality, i.e., $n_{P h}\left(L_{y}, d\right)<n_{Z h}\left(L_{y}, d\right)$, for all cases except $d=L_{y}$, where $n_{P h}\left(L_{y}, L_{y}\right)=1=n_{Z h}\left(L_{y}, L_{y}\right)$. We have calculated these $n_{P h}\left(L_{y}, d\right)$ and have obtained a number of interesting properties of the weighted graph coloring polynomial $\operatorname{Ph}\left(L_{y}, d\right)$. These are beyond the scope of the present work and hence will be presented elsewhere.

$$
\text { C. } q=0
$$

By substituting $q=0$ in (1.7) and noting the factorization $w^{n\left(G_{i}^{\prime}\right)}-1=(w-1) \sum_{\ell=0}^{n\left(G_{i}^{\prime}\right)-1} w^{\ell}$, we obtain the result that $Z(G, 0, v, w)$ contains a factor of $(w-1)$. 


\section{D. $q=1$}

Evaluating Eq. (1.1) for $q=1$, one sees that the Kronecker delta functions $\delta_{\sigma_{i} \sigma_{j}}=1$ for all pairs of adjacent vertices $\langle i, j\rangle$; consequently,

$$
Z(G, 1, v, w)=e^{K e(G)+h n(G)}=(v+1)^{e(G)} w^{n(G)}
$$

The coefficients $\tilde{c}^{(d)}$ evaluated at $q=1$ satisfy $[31]$

$$
\tilde{c}^{(d)}(q=1)=(-1)^{d}
$$

Hence, in terms of transfer matrices, we derive the following sum rule for the present cyclic lattice strips $G=\Lambda, L_{y} \times L_{x}, c y c$.

$$
\sum_{0 \leq d \leq L_{y}, d \text { even }} \operatorname{Tr}\left[\left(T_{Z, \Lambda, L_{y}, d}\right)^{m}\right]-\sum_{1 \leq d \leq L_{y}, d \text { odd }} \operatorname{Tr}\left[\left(T_{Z, \Lambda, L_{y}, d}\right)^{m}\right]=(v+1)^{e(G)} w^{n(G)} \text { for } q=1
$$

Here the number of edges $e(G)$ for each type of cyclic strip is

$$
e(G)= \begin{cases}\left(2 L_{y}-1\right) m & \text { if } \Lambda=s q \\ \left(3 L_{y}-2\right) m & \text { if } \Lambda=t r i \\ \left(3 L_{y}-1\right) m & \text { if } \Lambda=h c \\ 2 L_{y} m & \text { if } \Lambda=G_{D}\end{cases}
$$

where $m$ is given in terms of $L_{x}$ by Eq. (2.1). Since it applies for arbitrary $m$, the sum rule (3.7) implies relations between the eigenvalues of the various transfer matrices $T_{Z, \Lambda, L_{y}, d}$.

\section{E. $w=1$}

An interesting question concerns how $Z(G, q, v, w)$ reduces when $H \rightarrow 0$ (i.e., $w \rightarrow 1$ ). As will be evident from our explicit calculations, for generic $q$ and $v$, various $\lambda_{Z, L_{y}, d, j}$ 's in a given degree- $d$ subspace become equal to $\lambda_{Z, L_{y}, d^{\prime}, j}$ 's in a subspace of different degree, $d^{\prime}$. This process gives rise to "transmigration" of $\lambda_{Z, L_{y}, d, j}$ 's; as we combine the term(s) $\tilde{c}^{(d)}\left[\lambda_{Z, L_{y}, d^{\prime}, j}\right]^{m}$ from the degree- $d$ subspace(s) with the term $\tilde{c}^{\left(d^{\prime}\right)}\left[\lambda_{Z, L_{y}, d^{\prime}, j}\right]^{m}$ in the degree- $d^{\prime}$ subspace, this has the effect of yielding the zero-field term $c^{\left(d^{\prime}\right)}\left[\lambda_{Z, L_{y}, d^{\prime}, j}\right]^{m}$. Associated with this transmigration process, the $n_{Z h}\left(L_{y}, d\right)$ are changed to the $n_{Z}\left(L_{y}, d\right)$ given in Theorem 4 and Table 3 of [31] (see also [19]).

$$
\text { F. } w=0
$$

The special case $w=0$ is described by the relation (1.8), which is valid for an arbitrary graph $G$. From (1.4), it follows that $Z(G, q, v)$ contains a factor of $q$. From (1.8) it therefore follows that $Z(G, q, v, 0)$ contains a factor of $(q-1)$.

\section{GENERAL RESULTS FOR CYCLIC STRIPS OF THE SQUARE LATTICE}

In this section and the subsequent ones we present general results that we have obtained for the Potts model in an external magnetic field, valid for arbitrarily large strip width $L_{y}$ (as well as arbitrarily great length) for transfer matrices and their properties. We begin with strips of the square lattice. 


\section{A. Determinants}

We find

$$
\operatorname{det}\left(T_{Z, s q, L_{y}, d}\right)=\left(v^{L_{y}}\right)^{n_{Z h}\left(L_{y}, d\right)}\left[w^{L_{y}}\left(1+\frac{q}{v}\right)^{L_{y}}(1+v)^{L_{y}-1}\right]^{n_{Z h}\left(L_{y}-1, d\right)}
$$

where $n_{Z h}\left(L_{y}, d\right)$ was given in Theorem II.1. This applies for all $d$, i.e., $0 \leq d \leq L_{y}$ with $n_{Z h}\left(L_{y}-1, d\right)=0$ for $d>L_{y}-1$. The factor of $w$ in Eq. (4.1) originates from the diagonal matrix $K$ in Eq. (2.27), and the power of $w$ is the sum of the number of vertices in the $q=1$ state of all the $\left(L_{y}, d\right)$-partitions, which is the same as the power of $(1+q / v)$.

Next, taking into account that the generalized multiplicity of each $\lambda_{Z, s q, L_{y}, d}$ is $\tilde{c}^{(d)}$, we have, for the total determinant,

$$
\begin{aligned}
\operatorname{det}\left(T_{Z, s q, L_{y}}\right) & =\prod_{d=0}^{L_{y}}\left[\operatorname{det}\left(T_{\left.Z, s q, L_{y}, d\right)}\right]^{\tilde{c}^{(d)}}\right. \\
& =\prod_{d=0}^{L_{y}}\left[v^{L_{y}}\right]^{n_{Z h}\left(L_{y}, d\right) \tilde{c}^{(d)}}\left[w^{L_{y}}\left(1+\frac{q}{v}\right)^{L_{y}}(1+v)^{L_{y}-1}\right]^{n_{Z h}\left(L_{y}-1, d\right) \tilde{c}^{(d)}} \\
& =\left[v^{L_{y}}\right]^{\sum_{d=0}^{L_{y}} n_{Z h}\left(L_{y}, d\right) \tilde{c}^{(d)}}\left[w^{L_{y}}\left(1+\frac{q}{v}\right)^{L_{y}}(1+v)^{L_{y}-1}\right]^{\sum_{d=0}^{L_{y}} n_{Z h}\left(L_{y}-1, d\right) \tilde{c}^{(d)}}
\end{aligned}
$$

Using Eq. (2.14) together with $n_{Z h}\left(L_{y}, d\right)=0$ for $d>L_{y}$, so that $\sum_{d=0}^{L_{y}} n_{Z h}\left(L_{y}-1, d\right) \tilde{c}^{(d)}=\sum_{d=0}^{L_{y}-1} n_{Z h}\left(L_{y}-\right.$ $1, d) \tilde{c}^{(d)}$, we have

$$
\operatorname{det}\left(T_{Z, s q, L_{y}}\right)=\left[v^{L_{y}}\right]^{q^{L_{y}}}\left[w^{L_{y}}\left(1+\frac{q}{v}\right)^{L_{y}}(1+v)^{L_{y}-1}\right]^{q^{L_{y}-1}}
$$

This determinant of the transfer matrix for the cyclic strip of the square lattice applies for arbitrary width $L_{y}$. It is the generalization, to $H \neq 0$, of the zero-field result given in [29] with the extra $w$ factor.

\section{B. Eigenvalue for $d=L_{y}$ for $\Lambda=s q, t r i, h c$}

It was shown earlier that the $\lambda$ 's for the zero-field Potts model partition function are the same for a given lattice strip with cyclic, as compared with Möbius, boundary conditions [21, 36]. Indeed, this had been observed earlier for the special case of the chromatic polynomial, $v=-1$ [37]-[41] (and it was shown that the $\lambda$ 's for a strip with Klein bottle boundary conditions are a subset of the $\lambda$ 's for the same strip with torus boundary conditions [36, 42]). From Theorem II.1 one knows that there is only one $\lambda$ for degree $d=L_{y}$, which we denote as $\lambda_{Z, \Lambda, L_{y}, L_{y}}$. That is, for this value of $d$, the transfer matrix reduces to $1 \times 1$, i.e. a scalar. We found that for a cyclic or Möbius strip of the square, triangular, or honeycomb lattice with width $L_{y}$,

$$
\begin{gathered}
\lambda_{Z, \Lambda, L_{y}, L_{y}}=v^{L_{y}} \quad \text { for } \quad \Lambda=s q, \operatorname{tri} \\
\lambda_{Z, \Lambda, L_{y}, L_{y}}=v^{2 L_{y}} \quad \text { for } \quad \Lambda=h c
\end{gathered}
$$

which are the same as those for the zero-field case [29]. 


\section{Transfer Matrix for $d=L_{y}-1, \Lambda=s q$}

From Eq. (2.22) it follows that for $\Lambda=s q$, tri or $h c$, the number of $\lambda_{Z, \Lambda, L_{y}, d, j}$, for $d=L_{y}-1$ is $n_{Z h}\left(L_{y}, L_{y}-\right.$ $1)=3 L_{y}-1$, i.e. the transfer matrix in this subspace, $T_{Z, \Lambda, L_{y}, L_{y}-1}$, is a (square) $\left(3 L_{y}-1\right)$-dimensional matrix. For $L_{y}=1$,

$$
T_{Z, s q, 1,0}=\left(\begin{array}{cc}
v+q-1 & w \\
q-1 & w(1+v)
\end{array}\right)
$$

For $L_{y} \geq 2$ we find the following general formula.

$$
\begin{aligned}
& \left(T_{Z, s q, L_{y}, L_{y}-1}\right)_{j, j}=v^{L_{y}-1}(2 v+q-1) \quad \text { for } \quad j=1 \text { and } j=2 L_{y}-1 \\
& \left(T_{Z, s q, L_{y}, L_{y}-1}\right)_{2 j-1,2 j-1}=v^{L_{y}-1}(3 v+q-1) \quad \text { for } \quad L_{y} \geq 3 \quad \text { and } \quad 2 \leq j \leq L_{y}-1 \\
& \left(T_{Z, s q, L_{y}, L_{y}-1}\right)_{2 j, 2 j}=v^{L_{y}-1} w(1+v) \quad \text { for } \quad 1 \leq j \leq L_{y} \\
& \left(T_{Z, s q, L_{y}, L_{y}-1}\right)_{j+1, j}=v^{L_{y}-1}(v+q-1) \quad \text { for } \quad j=1 \text { and } j=2 L_{y}-1 \\
& \left(T_{Z, s q, L_{y}, L_{y}-1}\right)_{2 j, 2 j-1}=v^{L_{y}-1}(2 v+q-1) \quad \text { for } \quad L_{y} \geq 3 \quad \text { and } \quad 2 \leq j \leq L_{y}-1 \\
& \left(T_{Z, s q, L_{y}, L_{y}-1}\right)_{2 j-1,2 j}=v^{L_{y}-1} w \quad \text { for } \quad 1 \leq j \leq L_{y} \\
& \left(T_{Z, s q, L_{y}, L_{y}-1}\right)_{2 j-1,2 j+1}=\left(T_{Z, s q, L_{y}, L_{y}-1}\right)_{2 j, 2 j+1} \\
& =\left(T_{Z, s q, L_{y}, L_{y}-1}\right)_{2 j+1,2 j-1}=\left(T_{Z, s q, L_{y}, L_{y}-1}\right)_{2 j+2,2 j-1}=v^{L_{y}} \quad \text { for } \quad 1 \leq j \leq L_{y}-1 \\
& \left(T_{Z, s q, L_{y}, L_{y}-1}\right)_{j, j}=v^{L_{y}}(1+v) \quad \text { for } \quad 2 L_{y}+1 \leq j \leq 3 L_{y}-1 \\
& \left(T_{Z, s q, L_{y}, L_{y}-1}\right)_{2 j-1,2 L_{y}+j}=\left(T_{Z, s q, L_{y}, L_{y}-1}\right)_{2 j, 2 L_{y}+j} \\
& =\left(T_{Z, s q, L_{y}, L_{y}-1}\right)_{2 j+1,2 L_{y}+j}=\left(T_{Z, s q, L_{y}, L_{y}-1}\right)_{2 j+2,2 L_{y}+j}=v^{L_{y}-1}(1+v) \quad \text { for } \quad 1 \leq j \leq L_{y}-1 \\
& \left(T_{Z, s q, L_{y}, L_{y}-1}\right)_{2 L_{y}+j, 2 j-1}=\left(T_{Z, s q, L_{y}, L_{y}-1}\right)_{2 L_{y}+j, 2 j+1}=v^{L_{y}+1} \quad \text { for } \quad 1 \leq j \leq L_{y}-1
\end{aligned}
$$

with all other elements equal to zero. Thus, $T_{Z, s q, L_{y}, L_{y}-1}$ consists of four submatrices:

1. an upper left square submatrix with indices $i, j$ in the ranges $1 \leq i, j \leq 2 L_{y}$ and nonzero elements given by Eqs. (4.7)-(4.13)

2. a lower right square submatrix with indices in the ranges $2 L_{y}+1 \leq i, j \leq 3 L_{y}-1$ and nonzero elements given by Eqs. (4.14) 
3. an upper right rectangular submatrix with nonzero elements given by Eq. (4.15)

4. a lower left rectangular submatrix with nonzero elements given by Eq. 4.16)

For general $q, v$ and $w, T_{Z, s q, L_{y}, L_{y}-1}$ has rank equal to its dimension, $3 L_{y}-1$. We illustrate these general formulas for the cases $L_{y}=2$ and $L_{y}=3$. For this purpose we introduce the abbreviations

$$
v_{j}=j+v, \quad x_{j}=j v+q-1
$$

where $j$ is a positive integer. We have

$$
T_{Z, s q, 2,1}=v\left(\begin{array}{ccccc}
x_{2} & w & v & 0 & v_{1} \\
x_{1} & w v_{1} & v & 0 & v_{1} \\
v & 0 & x_{2} & w & v_{1} \\
v & 0 & x_{1} & w v_{1} & v_{1} \\
v^{2} & 0 & v^{2} & 0 & v v_{1}
\end{array}\right)
$$

and

$$
T_{Z, s q, 3,2}=v^{2}\left(\begin{array}{cccccccc}
x_{2} & w & v & 0 & 0 & 0 & v_{1} & 0 \\
x_{1} & w v_{1} & v & 0 & 0 & 0 & v_{1} & 0 \\
v & 0 & x_{3} & w & v & 0 & v_{1} & v_{1} \\
v & 0 & x_{2} & w v_{1} & v & 0 & v_{1} & v_{1} \\
0 & 0 & v & 0 & x_{2} & w & 0 & v_{1} \\
0 & 0 & v & 0 & x_{1} & w v_{1} & 0 & v_{1} \\
v^{2} & 0 & v^{2} & 0 & 0 & 0 & v v_{1} & 0 \\
0 & 0 & v^{2} & 0 & v^{2} & 0 & 0 & v v_{1}
\end{array}\right)
$$

In general, neglecting the $v^{L_{y}-1}$ factor, the upper left-hand submatrix has a main $2 \times 2$ block diagonal with end blocks equal to $\left(\begin{array}{cc}2 v+q-1 & w \\ v+q-1 & w(1+v)\end{array}\right)$ and interior blocks equal to $\left(\begin{array}{cc}3 v+q-1 & w \\ 2 v+q-1 & w(1+v)\end{array}\right)$. Adjacent to this main block diagonal are two block diagonals equal to $\left(\begin{array}{ll}v & 0 \\ v & 0\end{array}\right)$, and the rest of the submatrix is comprised of triangular regions filled with 0 's. The upper right-hand submatrix has a band of two $2 \times 1$ diagonals $\left(\begin{array}{c}1+v \\ 1+v\end{array}\right)$ together with triangular regions filled with 0's. The lower left-hand submatrix has a band of two $1 \times 2$ diagonals $\left(\begin{array}{ll}v^{2} & 0\end{array}\right)$ together with triangular regions filled with 0 's. And finally, in the right-hand lower submatrix the entries on the main diagonal are equal to $v(1+v)$ and the rest of this submatrix is made up of triangular regions of 0's. For the lowest values $L_{y}=1,2$, some of these parts, such as the triangular regions of zeros, are not present.

Having determined the general form of $T_{Z, s q, L_{y}, d}$ for $d=L_{y}-1$, we find that a pair of its eigenvalues $\lambda_{Z, s q, L_{y}, L_{y}-1, j}$ are roots of the following quadratic equation,

$$
x^{2}-v^{L_{y}-1}(v w+w+v+q-1) x+w v^{2 L_{y}-1}(v+q)=0
$$

As corollaries of our general result for $T_{Z, s q, L_{y}, L_{y}-1}$ we calculate the trace and determinant.

$$
\operatorname{det}\left(T_{Z, s q, L_{y}, L_{y}-1}\right)=v^{L_{y}\left(3 L_{y}-1\right)} w^{L_{y}}\left(1+\frac{q}{v}\right)^{L_{y}}(1+v)^{L_{y}-1}
$$

which is a special case of Eq. (4.1), and

$$
\operatorname{Tr}\left(T_{Z, s q, L_{y}, L_{y}-1}\right)=v^{L_{y}-1}\left[(q-1) L_{y}+v^{2}\left(L_{y}-1\right)+v\left(4 L_{y}-3\right)+w(1+v) L_{y}\right]
$$




\section{GENERAL RESULTS FOR CYCLIC STRIPS OF THE TRIANGULAR LATTICE}

\section{A. Determinants}

We find

$$
\operatorname{det}\left(T_{Z, t r i, L_{y}, d}\right)=\left(v^{L_{y}}\right)^{n_{Z h}\left(L_{y}, d\right)}\left[w^{L_{y}}\left(1+\frac{q}{v}\right)^{L_{y}}(1+v)^{2\left(L_{y}-1\right)}\right]^{n_{Z h}\left(L_{y}-1, d\right)}
$$

Comparing $V_{Z, s q, L_{y}, d}$ and $V_{Z, t r i, L_{y}, d}$ in Eq. (2.27), one sees that a set of $\left(I+v J_{L_{y}, d, i, i+1}\right)$ has been included for the triangular lattice, so that the power of $(1+v)$ becomes twice of the corresponding power for the square lattice.

Taking into account that the multiplicity of each $\lambda_{Z, t r i, L_{y}, d, j}$ is $\tilde{c}^{(d)}$, it follows that the total determinant is

$$
\operatorname{det}\left(T_{Z, t r i, L_{y}}\right) \equiv \prod_{d=0}^{L_{y}}\left[\operatorname{det}\left(T_{Z, t r i, L_{y}, d}\right)\right]^{\tilde{c}^{(d)}}=\left[v^{L_{y}}\right]^{q^{L_{y}}}\left[w^{L_{y}}\left(1+\frac{q}{v}\right)^{L_{y}}(1+v)^{2\left(L_{y}-1\right)}\right]^{q^{L_{y}-1}}
$$

\section{B. Transfer Matrix for $d=L_{y}-1, \Lambda=\operatorname{tri}$}

From Eq. (2.22), we know that the dimension of this transfer matrix is $3 L_{y}-1$ as before. The first nontrivial case is $L_{y}=2$. For $L_{y} \geq 2$ we find the following general formula.

$$
\begin{aligned}
& \left(T_{Z, t r i, L_{y}, L_{y}-1}\right)_{1,1}=v^{L_{y}-1}\left(v^{2}+4 v+q-1\right) \\
& \left(T_{Z, t r i, L_{y}, L_{y}-1}\right)_{2 j-1,2 j-1}=v^{L_{y}-1}\left(v^{2}+5 v+q-1\right) \quad \text { for } \quad 2 \leq j \leq L_{y}-1 \\
& \left(T_{Z, t r i, L_{y}, L_{y}-1}\right)_{2 L_{y}-1,2 L_{y}-1}=v^{L_{y}-1}(2 v+q-1) \\
& \left(T_{Z, t r i, L_{y}, L_{y}-1}\right)_{2 j, 2 j}=v^{L_{y}-1} w(1+v) \quad \text { for } \quad 1 \leq j \leq L_{y} \\
& \left(T_{Z, t r i, L_{y}, L_{y}-1}\right)_{2,1}=v^{L_{y}-1}\left(v^{2}+3 v+q-1\right) \\
& \left(T_{Z, t r i, L_{y}, L_{y}-1}\right)_{2 j, 2 j-1}=v^{L_{y}-1}\left(v^{2}+4 v+q-1\right) \quad \text { for } \quad 2 \leq j \leq L_{y}-1 \\
& \left(T_{Z, t r i, L_{y}, L_{y}-1}\right)_{2 L_{y}, 2 L_{y}-1}=v^{L_{y}-1}(v+q-1) \\
& \left(T_{Z, t r i, L_{y}, L_{y}-1}\right)_{2 j+1,2 j-1}=\left(T_{Z, t r i, L_{y}, L_{y}-1}\right)_{2 j+2,2 j-1}=v^{L_{y}} \quad \text { for } \quad 1 \leq j \leq L_{y}-1 \\
& \left(T_{Z, t r i, L_{y}, L_{y}-1}\right)_{j, k}=v^{L_{y}-1} w \quad \text { for } \quad k=2,4, \ldots, 2 L_{y} \quad \text { and } \quad 1 \leq j \leq k-1 \\
& \left(T_{Z, t r i, L_{y}, L_{y}-1}\right)_{j, k}=v^{L_{y}-1}\left(2 v^{2}+6 v+q-1\right) \quad \text { for } \quad k=3,5, \ldots, 2 L_{y}-3 \quad \text { and } \quad 1 \leq j \leq k-1 \\
& \left(T_{Z, t r i, L_{y}, L_{y}-1}\right)_{j, 2 L_{y}-1}=v^{L_{y}-1}\left(v^{2}+3 v+q-1\right) \quad \text { for } \quad 1 \leq j \leq 2 L_{y}-2
\end{aligned}
$$




$$
\begin{aligned}
& \left(T_{Z, t r i, L_{y}, L_{y}-1}\right)_{2 j+1,2 L_{y}+j}=\left(T_{Z, t r i, L_{y}, L_{y}-1}\right)_{2 j+2,2 L_{y}+j}=v^{L_{y}-1}(1+v) \quad \text { for } \quad 1 \leq j \leq L_{y}-1 \\
& \left(T_{Z, t r i, L_{y}, L_{y}-1}\right)_{j, 2 L_{y}+k}=v^{L_{y}-1}(v+1)(v+2) \quad \text { for } \quad 1 \leq k \leq L_{y}-1 \quad \text { and } \quad 1 \leq j \leq 2 k \\
& \left(T_{Z, t r i, L_{y}, L_{y}-1}\right)_{2 L_{y}+j, 2 j-1}=v^{L_{y}+1}(3+v) \quad \text { for } \quad 1 \leq j \leq L_{y}-1 \\
& \left(T_{Z, t r i, L_{y}, L_{y}-1}\right)_{2 L_{y}+j, 2 k-1}=v^{L_{y}}\left(2 v^{2}+6 v+q-1\right) \quad \text { for } \quad 2 \leq k \leq L_{y}-1 \quad \text { and } \quad 1 \leq j \leq k-1 \\
& \left(T_{Z, t r i, L_{y}, L_{y}-1}\right)_{2 L_{y}+j, 2 L_{y}-1}=v^{L_{y}}\left(v^{2}+3 v+q-1\right) \quad \text { for } \quad 1 \leq j \leq L_{y}-1 \\
& \left(T_{Z, t r i, L_{y}, L_{y}-1}\right)_{2 L_{y}+j, k}=v^{L_{y}} w \quad \text { for } \quad k=4,6, \ldots, 2 L_{y} \quad \text { and } \quad 1 \leq j \leq k / 2-1 \\
& \left(T_{Z, t r i, L_{y}, L_{y}-1}\right)_{2 L_{y}+j, 2 L_{y}+k}=v^{L_{y}}(v+1)(v+2) \quad \text { for } \quad 1 \leq j \leq k \leq L_{y}-1
\end{aligned}
$$

with all other elements equal to zero. Thus, $T_{Z, t r i, L_{y}, L_{y}-1}$ can again be usefully viewed as consisting of various submatrices.

For general $q, v$ and $w, T_{Z, t r i, L_{y}, L_{y}-1}$ has rank equal to its dimension, $3 L_{y}-1$. We illustrate these general formulas with some explicit examples for $L_{y}=2$ and $L_{y}=3$. For compactness of notation, we use the abbreviations

$$
y_{j}=v^{2}+j v+q-1, \quad z_{j}=2 v^{2}+j v+q-1
$$

where $j$ is a positive integer. Then

$$
\begin{aligned}
& T_{Z, t r i, 2,1}=v\left(\begin{array}{ccccc}
y_{4} & w & y_{3} & w & v_{1} v_{2} \\
y_{3} & w v_{1} & y_{3} & w & v_{1} v_{2} \\
v & 0 & x_{2} & w & v_{1} \\
v & 0 & x_{1} & w v_{1} & v_{1} \\
v^{2} v_{3} & 0 & v y_{3} & w v & v v_{1} v_{2}
\end{array}\right) \\
& T_{Z, t r i, 3,2}=v^{2}\left(\begin{array}{cccccccc}
y_{4} & w & z_{6} & w & y_{3} & w & v_{1} v_{2} & v_{1} v_{2} \\
y_{3} & w v_{1} & z_{6} & w & y_{3} & w & v_{1} v_{2} & v_{1} v_{2} \\
v & 0 & y_{5} & w & y_{3} & w & v_{1} & v_{1} v_{2} \\
v & 0 & y_{4} & w v_{1} & y_{3} & w & v_{1} & v_{1} v_{2} \\
0 & 0 & v & 0 & x_{2} & w & 0 & v_{1} \\
0 & 0 & v & 0 & x_{1} & w v_{1} & 0 & v_{1} \\
v^{2} v_{3} & 0 & v z_{6} & w v & v y_{3} & w v & v v_{1} v_{2} & v v_{1} v_{2} \\
0 & 0 & v^{2} v_{3} & 0 & v y_{3} & w v & 0 & v v_{1} v_{2}
\end{array}\right)
\end{aligned}
$$

As corollaries of our general result for $T_{Z, t r i, L_{y}, L_{y}-1}$ we calculate the trace and determinant.

$$
\operatorname{det}\left(T_{Z, t r i, L_{y}, L_{y}-1}\right)=v^{L_{y}\left(3 L_{y}-1\right)} w^{L_{y}}\left(1+\frac{q}{v}\right)^{L_{y}}(1+v)^{2\left(L_{y}-1\right)}
$$

which is a special case of Eq. (5.1), and

$$
\operatorname{Tr}\left(T_{Z, t r i, L_{y}, L_{y}-1}\right)=v^{L_{y}-1}\left[(q-1) L_{y}+v^{3}\left(L_{y}-1\right)+4 v^{2}\left(L_{y}-1\right)+v\left(7 L_{y}-6\right)+w(1+v) L_{y}\right]
$$




\section{GENERAL RESULTS FOR CYCLIC STRIPS OF THE HONEYCOMB LATTICE}

\section{A. Determinants}

We find

$$
\operatorname{det}\left(T_{Z, h c, L_{y}, d}\right)=\left(v^{2 L_{y}}\right)^{n_{Z h}\left(L_{y}, d\right)}\left[w^{2 L_{y}}\left(1+\frac{q}{v}\right)^{2 L_{y}}(1+v)^{L_{y}-1}\right]^{n_{Z h}\left(L_{y}-1, d\right)}
$$

This can be understood as follows: by an argument similar to that given before, the power of $(1+v)$ is the same as for the square lattice case. Comparing $T_{Z, s q, L_{y}, d}$ and $T_{Z, h c, L_{y}, d}$ in Eq. (2.28), one sees that $V_{Z, h c, L_{y}, d}=V_{Z, s q, L_{y}, d}$ has been multiplied twice for the honeycomb lattice, so that the powers of $v$ and $(1+q / v)$ become twice of the corresponding powers for the square lattice. In Eq. (2.27), both $H_{Z, h c, L_{y}, d, 1}$ and $H_{Z, h c, L_{y}, d, 2}$ include the matrix $K$ so that the powers of $w$ also become twice of the corresponding powers for the square lattice.

Taking into account that the multiplicity of each $\lambda_{Z, h c, L_{y}, d, j}$ is $\tilde{c}^{(d)}$, it follows that the total determinant for the $h c$ lattice is

$$
\operatorname{det}\left(T_{Z, h c, L_{y}}\right) \equiv \prod_{d=0}^{L_{y}}\left[\operatorname{det}\left(T_{Z, h c, L_{y}, d}\right)\right]^{\tilde{c}^{(d)}}=\left(v^{2 L_{y}}\right)^{q^{L_{y}}}\left[w^{2 L_{y}}\left(1+\frac{q}{v}\right)^{2 L_{y}}(1+v)^{L_{y}-1}\right]^{q^{L_{y}-1}}
$$

Summarizing the connections between the determinants of the transfer matrices for the three lattice strips, $\operatorname{det}\left(T_{Z, t r i, L_{y}, d}\right)$ is related to $\operatorname{det}\left(T_{Z, s q, L_{y}, d}\right)$ by the replacement of $(1+v)$ by $(1+v)^{2}$ and $\operatorname{det}\left(T_{Z, h c, L_{y}, d}\right)$ is related to $\operatorname{det}\left(T_{Z, s q, L_{y}, d}\right)$ by the replacements of the respective factors $w$ by $w^{2}, v$ by $v^{2}$ and $\left(1+\frac{q}{v}\right)$ by $\left(1+\frac{q}{v}\right)^{2}$. This, together with the fact that $n_{Z h}\left(L_{y}, d\right)$ is the same for all of these three lattices means that the total determinants $\operatorname{det}\left(T_{Z, t r i, L_{y}}\right)$ and $\operatorname{det}\left(T_{Z, h c, L_{y}}\right)$ are related to $\operatorname{det}\left(T_{Z, s q, L_{y}}\right)$ by the same respective replacements.

\section{B. Transfer Matrix for $d=L_{y}-1, \Lambda=h c$}

From Eq. (2.22), we know that the dimension of this transfer matrix is again $3 L_{y}-1$. The first nontrivial case is $L_{y}=2$. Recall in Eq. (2.28) the transfer matrix for the honeycomb lattice, $T_{Z, h c, L_{y}, L_{y}-1}$, is the product of $T_{Z, h c, L_{y}, L_{y}-1,1}$ and $T_{Z, h c, L_{y}, L_{y}-1,2}$. For $L_{y} \geq 2$ we find the following general formula.

$$
\begin{aligned}
& \left(T_{Z, h c, L_{y}, L_{y}-1,1}\right)_{2 j-1,2 j-1}=v^{L_{y}-1}(2 v+q-1) \quad \text { for } \quad 1 \leq j \leq L_{y}-1 \\
& \left(T_{Z, h c, L_{y}, L_{y}-1,1}\right)_{2 L_{y}-1,2 L_{y}-1}= \begin{cases}v^{L_{y}-1}(2 v+q-1) & \text { for } L_{y} \text { even } \\
v^{L_{y}-1}(v+q-1) & \text { for } L_{y} \text { odd }\end{cases} \\
& \left(T_{Z, h c, L_{y}, L_{y}-1,1}\right)_{2 j, 2 j}=v^{L_{y}-1} w(1+v) \quad \text { for } \quad 1 \leq j \leq L_{y} \\
& \left(T_{Z, h c, L_{y}, L_{y}-1,1}\right)_{2 j, 2 j-1}=v^{L_{y}-1}(v+q-1) \quad \text { for } \quad 1 \leq j \leq L_{y}-1 \\
& \left(T_{Z, h c, L_{y}, L_{y}-1,1}\right)_{2 L_{y}, 2 L_{y}-1}= \begin{cases}v^{L_{y}-1}(v+q-1) & \text { for } L_{y} \text { even } \\
v^{L_{y}-1}(q-1) & \text { for } L_{y} \text { odd }\end{cases} \\
& \left(T_{Z, h c, L_{y}, L_{y}-1,1}\right)_{2 j-1,2 j}=v^{L_{y}-1} w \quad \text { for } \quad 1 \leq j \leq L_{y}
\end{aligned}
$$




$$
\begin{aligned}
& \left(T_{Z, h c, L_{y}, L_{y}-1,1}\right)_{4 j-1,4 j-3}=\left(T_{Z, h c, L_{y}, L_{y}-1,1}\right)_{4 j, 4 j-3} \\
& =\left(T_{Z, h c, L_{y}, L_{y}-1,1}\right)_{4 j-3,4 j-1}=\left(T_{Z, h c, L_{y}, L_{y}-1,1}\right)_{4 j-2,4 j-1}=v^{L_{y}} \quad \text { for } \quad 1 \leq j \leq\left[L_{y} / 2\right] \\
& \left(T_{Z, h c, L_{y}, L_{y}-1,1}\right)_{2 L_{y}+2 j-1,2 L_{y}+2 j-1}=v^{L_{y}}(1+v) \quad \text { for } \quad 1 \leq j \leq\left[L_{y} / 2\right] \\
& \left(T_{Z, h c, L_{y}, L_{y}-1,1}\right)_{2 L_{y}+2 j, 2 L_{y}+2 j}=v^{L_{y}} \quad \text { for } \quad 1 \leq j \leq\left[\left(L_{y}-1\right) / 2\right] \\
& \left(T_{Z, h c, L_{y}, L_{y}-1,1}\right)_{4 j-3,2 L_{y}+2 j-1}=\left(T_{Z, h c, L_{y}, L_{y}-1,1}\right)_{4 j-2,2 L_{y}+2 j-1} \\
& =\left(T_{Z, h c, L_{y}, L_{y}-1,1}\right)_{4 j-1,2 L_{y}+2 j-1}=\left(T_{Z, h c, L_{y}, L_{y}-1,1}\right)_{4 j, 2 L_{y}+2 j-1} \\
& =v^{L_{y}-1}(v+1) \text { for } 1 \leq j \leq\left[L_{y} / 2\right] \\
& \left(T_{Z, h c, L_{y}, L_{y}-1,1}\right)_{4 j-1,2 L_{y}+2 j}=\left(T_{Z, h c, L_{y}, L_{y}-1,1}\right)_{4 j, 2 L_{y}+2 j} \\
& =\left(T_{Z, h c, L_{y}, L_{y}-1,1}\right)_{4 j+1,2 L_{y}+2 j}=\left(T_{Z, h c, L_{y}, L_{y}-1,1}\right)_{4 j+2,2 L_{y}+2 j} \\
& =v^{L_{y}-1} \text { for } 1 \leq j \leq\left[\left(L_{y}-1\right) / 2\right] \\
& \left(T_{Z, h c, L_{y}, L_{y}-1,2}\right)_{2 L_{y}+2 j-1,2 L_{y}+2 j-1}=v^{L_{y}} \quad \text { for } \quad 1 \leq j \leq\left[L_{y} / 2\right]
\end{aligned}
$$




$$
\begin{aligned}
& \left(T_{Z, h c, L_{y}, L_{y}-1,2}\right)_{2 L_{y}+2 j, 2 L_{y}+2 j}=v^{L_{y}}(1+v) \quad \text { for } \quad 1 \leq j \leq\left[\left(L_{y}-1\right) / 2\right] \\
& \left(T_{Z, h c, L_{y}, L_{y}-1,2}\right)_{4 j-3,2 L_{y}+2 j-1}=\left(T_{Z, h c, L_{y}, L_{y}-1,2}\right)_{4 j-2,2 L_{y}+2 j-1} \\
& =\left(T_{Z, h c, L_{y}, L_{y}-1,2}\right)_{4 j-1,2 L_{y}+2 j-1}=\left(T_{Z, h c, L_{y}, L_{y}-1,2}\right)_{4 j, 2 L_{y}+2 j-1} \\
& =v^{L_{y}-1} \quad \text { for } \quad 1 \leq j \leq\left[L_{y} / 2\right] \\
& \left(T_{Z, h c, L_{y}, L_{y}-1,2}\right)_{4 j-1,2 L_{y}+2 j}=\left(T_{Z, h c, L_{y}, L_{y}-1,2}\right)_{4 j, 2 L_{y}+2 j} \\
& =\left(T_{Z, h c, L_{y}, L_{y}-1,2}\right)_{4 j+1,2 L_{y}+2 j}=\left(T_{Z, h c, L_{y}, L_{y}-1,2}\right)_{4 j+2,2 L_{y}+2 j} \\
& =v^{L_{y}-1}(1+v) \text { for } 1 \leq j \leq\left[\left(L_{y}-1\right) / 2\right]
\end{aligned}
$$

with all other elements equal to zero.

For general $q, v$ and $w, T_{Z, h c, L_{y}, L_{y}-1}$ has rank equal to its dimension, $3 L_{y}-1$. We illustrate these general formulas for the cases $L_{y}=2$ and $L_{y}=3$ (with the same abbreviations as before):

$$
\begin{gathered}
T_{Z, h c, 2,1,1}=T_{Z, s q, 2,1}, \quad T_{Z, h c, 2,1,2}=v\left(\begin{array}{cccccc}
x_{1} & w & 0 & 0 & 1 \\
q-1 & w v_{1} & 0 & 0 & 1 \\
0 & 0 & x_{1} & w & 1 \\
0 & 0 & q-1 & w v_{1} & 1 \\
0 & 0 & 0 & 0 & v
\end{array}\right) \\
T_{Z, h c, 3,2,1}=v^{2}\left(\begin{array}{cccccccc}
x_{2} & w & v & 0 & 0 & 0 & v_{1} & 0 \\
x_{1} & w v_{1} & v & 0 & 0 & 0 & v_{1} & 0 \\
v & 0 & x_{2} & w & 0 & 0 & v_{1} & 1 \\
v & 0 & x_{1} & w v_{1} & 0 & 0 & v_{1} & 1 \\
0 & 0 & 0 & 0 & x_{1} & w & 0 & 1 \\
0 & 0 & 0 & 0 & q-1 & w v_{1} & 0 & 1 \\
v^{2} & 0 & v^{2} & 0 & 0 & 0 & v v_{1} & 0 \\
0 & 0 & 0 & 0 & 0 & 0 & 0 & v
\end{array}\right) \\
T_{Z, h c, 3,2,2}=v^{2}\left(\begin{array}{cccccccc}
x_{1} & w & 0 & 0 & 0 & 0 & 1 & 0 \\
q-1 & w v_{1} & 0 & 0 & 0 & 0 & 1 & 0 \\
0 & 0 & x_{2} & w & v & 0 & 1 & v_{1} \\
0 & 0 & x_{1} & w v_{1} & v & 0 & 1 & v_{1} \\
0 & 0 & v & 0 & x_{2} & w & 0 & v_{1} \\
0 & 0 & v & 0 & x_{1} & w v_{1} & 0 & v_{1} \\
0 & 0 & 0 & 0 & 0 & 0 & v & 0 \\
0 & 0 & v^{2} & 0 & v^{2} & 0 & 0 & v v_{1}
\end{array}\right)
\end{gathered}
$$

The $T_{Z, h c, L_{y}, L_{y}-1}$ are obtained via Eq. (2.28) from these auxiliary matrices. For example,

$$
=v^{2}\left(\begin{array}{ccccc}
x_{1}(3 v+w)+(q-1)^{2} & w\left(x_{1}+w v_{1}\right) & v\left(x_{2}+w\right) & 0 & v_{1}\left(x_{2}+w\right) \\
x_{1}\left(x_{1}+w v_{1}\right) & w\left(w v_{1}^{2}+q-1\right) & v\left(x_{1}+w v_{1}\right) & 0 & v_{1}\left(x_{1}+w v_{1}\right) \\
v\left(x_{2}+w\right) & 0 & x_{1}(3 v+w)+(q-1)^{2} & w\left(x_{1}+w v_{1}\right) & v_{1}\left(x_{2}+w\right) \\
v\left(x_{1}+w v_{1}\right) & 0 & x_{1}\left(x_{1}+w v_{1}\right) & w\left(w v_{1}^{2}+q-1\right) & v_{1}\left(x_{1}+w v_{1}\right) \\
v^{3} & 0 & v^{3} & 0 & v^{2} v_{1}
\end{array}\right)
$$


We find that in general, for degree $d=L_{y}-1$, a pair of its eigenvalues $\lambda_{Z, h c, L_{y}, L_{y}-1, j}$ are roots of the following quadratic equation,

$$
x^{2}-v^{2 L_{y}-2}\left(w^{2}(v+1)^{2}+2(q-1)(v+w)+(q-1)^{2}+v^{2}\right) x+w^{2} v^{4 L_{y}-2}(v+q)^{2}=0
$$

The expressions for the other $3 L_{y}-3$ eigenvalues are, in general, more complicated.

\section{GENERAL RESULTS FOR CYCLIC SELF-DUAL SQUARE-LATTICE STRIPS}

In this section we consider the Potts model for families of self-dual strip graphs of the square lattice with fixed width $L_{y}$ and arbitrarily great length $L_{x}$, having periodic longitudinal boundary conditions, such that all vertices on one side of the strip, which we take to be the upper side, are joined by edges to a single external vertex. A strip graph of this type will be denoted generically as $G_{D}$ and, in more detail, as $G_{D}\left(L_{y} \times L_{x}\right)$. The family of $G_{D}$ graphs is planar and self-dual. We recall that for a planar graph $G_{p l}$, one defines the (planar) dual graph $G_{p l}^{*}$ as the graph obtained by replacing each vertex (face) of $G_{p l}$ by a face (vertex) of $G_{p l}^{*}$ and connecting the vertices of the resultant $G_{p l}^{*}$ by edges when the corresponding faces of $G_{p l}$ have a common edge. The graph $G_{p l}$ is self-dual if and only if $G_{p l}=G_{p l}^{*}$. For zero-field, it is known that

$$
Z\left(G_{p l}, q, v, w=1\right)=v^{e\left(G_{p l}\right)} q^{-c\left(G_{p l}\right)} Z\left(G_{p l}^{*}, q, \frac{q}{v}, w=1\right)
$$

for a planar graph $G_{p l}$. In general, the graph $G_{D}\left(L_{y} \times L_{x}\right)$ has $n \equiv|V|=L_{x} L_{y}+1$ vertices, equal to the number of faces, $f$. One motivation for considering the $G_{D}$ strip graphs is that they exhibit, for any $L_{y}$, the self-duality property of the infinite square lattice so that the zero-field partition function is invariant under $v \rightarrow q / v$ by (7.1), aside from a prefactor.

In Ref. [32] we gave the general form for the zero-field Potts model partition function $Z\left(G_{D}, L_{y} \times L_{x}, q, v, w=\right.$ 1). In our current notation with $m$ given in terms of $L_{x}$ by Eq. (2.1), this is

$$
Z\left(G_{D}, L_{y} \times L_{x}, q, v, 1\right)=\sum_{d=1}^{L_{y}+1} \kappa^{(d)} \operatorname{Tr}\left[\left(T_{Z, G_{D}, L_{y}, d}\right)^{m}\right]
$$

where

$$
\kappa^{(d)}=\sqrt{q} U_{2 d-1}\left(\frac{\sqrt{q}}{2}\right)=\sum_{j=0}^{d-1}(-1)^{j}\left(\begin{array}{c}
2 d-1-j \\
j
\end{array}\right) q^{d-j}
$$

To construct the transfer matrix $T_{Z, G_{D}, L_{y}, d}$ for each $d$, we begin with partitions with $L_{y}+1$ vertices, where the single external vertex is considered as the $\left(L_{y}+1\right)$-th vertex, so that the size of the matrix is $n_{Z h}\left(L_{y}+1, d\right)$ with $0 \leq d \leq L_{y}+1$. When the field is non-zero, the transverse and longitudinal parts, $H_{Z, G_{D}, L_{y}, d}$ and $V_{Z, G_{D}, L_{y}, d}$, of the transfer matrix $T_{Z, G_{D}, L_{y}, d}$ can be expressed as

$$
\begin{aligned}
H_{Z, G_{D}, L_{y}, d} & =K \prod_{i=1}^{L_{y}}\left(I+v J_{L_{y}, d, i, i+1}\right), \quad \bar{H}_{Z, G_{D}, L_{y}, d}=\bar{K} \prod_{i=1}^{L_{y}}\left(I+v J_{L_{y}, d, i, i+1}\right) \\
V_{Z, G_{D}, L_{y}, d} & =\prod_{i=1}^{L_{y}}\left(v I+D_{L_{y}, d, i}\right) \\
T_{Z, G_{D}, L_{y}, d} & =V_{Z, G_{D}, L_{y}, d} H_{Z, G_{D}, L_{y}, d}, \quad \bar{T}_{Z, G_{D}, L_{y}, d}=V_{Z, G_{D}, L_{y}, d} \bar{H}_{Z, G_{D}, L_{y}, d}
\end{aligned}
$$

where $K$ is again the diagonal matrix with diagonal element $w^{\ell}$ where $\ell$ is the number of vertices in the $q=1$ state for the corresponding basis, and $\bar{K}$ is the diagonal matrix with diagonal element $w^{\bar{\ell}}$ where $\bar{\ell}$ is 
the number of vertices, excluding the $\left(L_{y}+1\right)$-th vertex, in the $q=1$ state. Notice that $V_{Z, G_{D}, L_{y}, d}$ does not include the factor $\left(v I+D_{L_{y}, d, L_{y}+1}\right)$ for the single external vertex. We have

$$
Z\left(G_{D}, L_{y} \times L_{x}, q, v, w\right)=\sum_{d=0}^{L_{y}+1} \tilde{c}^{(d)} \operatorname{Tr}\left[T_{Z, G_{D}, L_{y}, d}\left(\bar{T}_{Z, G_{D}, L_{y}, d}\right)^{m-1}\right]
$$

Here only one $T_{Z, G_{D}, L_{y}, d}$ is needed as the single external vertex in the $q=1$ state should only be considered once. Compare matrices $T_{Z, G_{D}, L_{y}, d}$ and $\bar{T}_{Z, G_{D}, L_{y}, d}$, certain columns differ by a factor of $w$, corresponding to the partitions with the $\left(L_{y}+1\right)$-th vertex in the $q=1$ state. It is clear that the number of these columns is $n_{Z h}\left(L_{y}, d\right)$, i.e. the cross case in category (b) in the proof of Theorem II.1 It follows that there are $n_{Z h}\left(L_{y}, d\right)$ eigenvalues of $T_{Z, G_{D}, L_{y}, d}$ equal to the corresponding eigenvalues of $\bar{T}_{Z, G_{D}, L_{y}, d}$ multiplied by $w$, and the rest of the eigenvalues are the same. Let us denote the eigenvalues that are common to $T_{Z, G_{D}, L_{y}, d}$ and $\bar{T}_{Z, G_{D}, L_{y}, d}$ as usual as $\lambda_{Z, G_{D}, L_{y}, d, j}$, and the eigenvalues of $\bar{T}_{Z, G_{D}, L_{y}, d}$ that lack a factor of $w$ as $\bar{\lambda}_{Z, G_{D}, L_{y}, d, j}$.

Furthermore, the common eigenvalues $\lambda_{Z, G_{D}, L_{y}, d, j}$ at level $d$ also appear as $\lambda_{Z, G_{D}, L_{y}, d+1, j}$ at level $d+1$ with $0 \leq d \leq L_{y}$. This again is due to the fact that $V_{Z, G_{D}, L_{y}, d}$ does not include the factor $\left(v I+D_{L_{y}, d, L_{y}+1}\right)$, so that it does not matter if the $\left(L_{y}+1\right)$-th vertex is assigned a color (connected to a black circle) or not. Denote the number of these common eigenvalues as $n_{Z h}\left(G_{D}, L_{y}, d\right)$ with $1 \leq d \leq L_{y}+1$. They are given by

$$
n_{Z h}\left(G_{D}, L_{y}, d\right)=n_{Z h}\left(L_{y}, d-1\right)+n_{Z h}\left(L_{y}, d\right)
$$

That is, they can be classified as being in either category (a) plus category (c) in the proof of Theorem II.1, or the circle case in category (b) plus category (d) with $d$ replaced by $d-1$ in that proof. In Table $\nabla$ we list the first few numbers $n_{Z h}\left(G_{D}, L_{y}, d\right)$ and their total sums $N_{Z h, G_{D}, L_{y}}$. In particular, the numbers $n_{Z h}\left(G_{D}, L_{y}, 1\right)$ is

$$
\begin{gathered}
n_{Z h}\left(G_{D}, L_{y}, 1\right)=\sum_{k=1}^{L_{y}+1}\left(\begin{array}{c}
L_{y} \\
k-1
\end{array}\right) C_{k} \\
n_{Z h}\left(G_{D}, L_{y}, L_{y}\right)=3 L_{y}, \quad n_{Z h}\left(G_{D}, L_{y}, L_{y}+1\right)=1
\end{gathered}
$$

If we denote the generating function of $n_{Z h}\left(G_{D}, L_{y}, 1\right)$ as

$$
A_{1}(x)=\sum_{L_{y}=0}^{\infty} n_{Z h}\left(G_{D}, L_{y}, 1\right) x^{L_{y}+1}=x+3 x^{2}+10 x^{3}+36 x^{4}+\ldots
$$

then the generating function of $n_{Z h}\left(G_{D}, L_{y}, 2\right)$, denoted as $A_{2}(x)$, is given by the convolution [34]

$$
A_{2}(x)=\sum_{L_{y}=1}^{\infty} n_{Z h}\left(G_{D}, L_{y}, 2\right) x^{L_{y}+1}=\left[A_{1}(x)\right]^{2}=x^{2}+6 x^{3}+29 x^{4}+132 x^{5}+\ldots
$$

In general, the generating function of $n_{Z h}\left(G_{D}, L_{y}, d\right)$ is given as

$$
A_{d}(x)=\sum_{L_{y}=d-1}^{\infty} n_{Z h}\left(G_{D}, L_{y}, d\right) x^{L_{y}+1}=\left[A_{1}(x)\right]^{d}
$$

From Eq. (7.6), it is clear that

$$
N_{Z h, G_{D}, L_{y}}=2 N_{Z h, L_{y}}-n_{Z h}\left(L_{y}, 0\right)
$$

It follows that

$$
N_{Z h, G_{D}, L_{y}+1}=5 N_{Z h, G_{D}, L_{y}}-n_{Z h}\left(G_{D}, L_{y}, 1\right)
$$


TABLE V: Table of numbers $n_{Z h}\left(L_{y}, G_{D}, d\right)$ and their sums, $N_{Z h, G_{D}, L_{y}}$. Blank entries are zero.

\begin{tabular}{|c|c|c|c|c|c|c|c|c|c|c|c|}
\hline \hline$L_{y} \backslash d$ & 1 & 2 & 3 & 4 & 5 & 6 & 7 & 8 & 9 & 10 & $N_{Z h, G_{D}, L_{y}}$ \\
\hline \hline 1 & 3 & 1 & & & & & & & & & 4 \\
\hline 2 & 10 & 6 & 1 & & & & & & & & 17 \\
\hline 3 & 36 & 29 & 9 & 1 & & & & & & & 75 \\
\hline 4 & 137 & 132 & 57 & 12 & 1 & & & & & & 339 \\
\hline 5 & 543 & 590 & 315 & 94 & 15 & 1 & & & & & 1558 \\
\hline 6 & 2219 & 2628 & 1629 & 612 & 140 & 18 & 1 & & & & 7247 \\
\hline 7 & 9285 & 11732 & 8127 & 3605 & 1050 & 195 & 21 & 1 & & & 34016 \\
\hline 8 & 39587 & 52608 & 39718 & 19992 & 6950 & 1656 & 259 & 24 & 1 & & 160795 \\
\hline 9 & 171369 & 237129 & 191754 & 106644 & 42498 & 12177 & 2457 & 332 & 27 & 1 & 764388 \\
\hline \hline
\end{tabular}

similar to Eq. (2.23), and $N_{Z h, G_{D}, L_{y}}$ can be expressed as

$$
N_{Z h, G_{D}, L_{y}}=\sum_{j=0}^{L_{y}}\left(\begin{array}{c}
L_{y} \\
j
\end{array}\right)\left(\begin{array}{c}
2 j+1 \\
j+1
\end{array}\right)
$$

The multiplicity for the common eigenvalues with $1 \leq d$ is defined as

$$
\tilde{\kappa}^{(d)}(q)=\kappa^{(d)}(q-1)=\tilde{c}^{(d)}+\tilde{c}^{(d-1)}=\sum_{j=0}^{d-1}(-1)^{j}\left(\begin{array}{c}
2 d-1-j \\
j
\end{array}\right)(q-1)^{d-j}
$$

where $\kappa^{(d)}(q)$ is given in Eq. (7.3). The first few of these coefficients are

$$
\begin{aligned}
& \tilde{\kappa}^{(1)}=q-1, \quad \tilde{\kappa}^{(2)}=(q-1)(q-3), \\
& \tilde{\kappa}^{(3)}=(q-1)(q-2)(q-4), \quad \tilde{\kappa}^{(4)}=(q-1)(q-3)\left(q^{2}-6 q+7\right)
\end{aligned}
$$

Collecting the common eigenvalues, we find that the partition function in Eq. (7.5) can be rewritten as

$$
\begin{aligned}
Z\left(G_{D}, L_{y} \times L_{x}, q, v, w\right)= & \sum_{d=1}^{L_{y}+1} \tilde{\kappa}^{(d)} \sum_{j=1}^{n_{Z h}\left(L_{y}, G_{D}, d\right)}\left(\lambda_{Z, G_{D}, L_{y}, d, j}\right)^{m} \\
& +w \sum_{d=0}^{L_{y}} \tilde{c}^{(d)} \sum_{j=1}^{n_{Z h}\left(L_{y}, d\right)}\left(\bar{\lambda}_{Z, G_{D}, L_{y}, d, j}\right)^{m}
\end{aligned}
$$

where $n_{Z h}\left(L_{y}, d\right)$ is given in Theorem $\amalg .1$

\section{A. Determinants}

We find

$$
\begin{aligned}
\operatorname{det}\left(T_{Z, G_{D}, L_{y}, d}\right) & =\left(v^{L_{y}}\right)^{n_{Z h}\left(L_{y}+1, d\right)}\left[w^{L_{y}+1}\left(1+\frac{q}{v}\right)^{L_{y}}(1+v)^{L_{y}}\right]^{n_{Z h}\left(L_{y}, d\right)} \\
\operatorname{det}\left(\bar{T}_{Z, G_{D}, L_{y}, d}\right) & =\left(v^{L_{y}}\right)^{n_{Z h}\left(L_{y}+1, d\right)}\left[w\left(1+\frac{q}{v}\right)(1+v)\right]^{L_{y} n_{Z h}\left(L_{y}, d\right)}
\end{aligned}
$$


for the full range $0 \leq d \leq L_{y}+1$. Comparing $V_{Z, s q, L_{y}+1, d}$ in Eq. (2.27) and $V_{Z, G_{D}, L_{y}, d}$ in Eq. (7.4), one sees that $V_{Z, G_{D}, L_{y}, d}$ does not include the factor $\left(v I+D_{L_{y}, d, L_{y}+1}\right)$ for the self-dual square lattice, so that the power of $v$ and $(1+q / v)$ has the factor $L_{y}$ rather than $L_{y}+1$. Comparing $H_{Z, G_{D}, L_{y}, d}$ and $\bar{H}_{Z, G_{D}, L_{y}, d}$ in Eq. (17.4), one sees that the power of $w$ for $\bar{H}_{Z, G_{D}, L_{y}, d}$ has the factor $L_{y}$ rather than $L_{y}+1$ in $H_{Z, G_{D}, L_{y}, d}$.

Taking into account that the multiplicity of each $\lambda_{Z, G_{D}, L_{y}, d, j}$ is $\tilde{c}^{(d)}$, it follows that the total determinant is

$$
\operatorname{det}\left(T_{Z, G_{D}, L_{y}}\right) \equiv \prod_{d=0}^{L_{y}+1}\left[\operatorname{det}\left(T_{Z, G_{D}, L_{y}, d}\right)\right]^{\tilde{c}^{(d)}}=\left[v^{L_{y}}\right]^{q^{L_{y}+1}}\left[w^{L_{y}+1}\left(1+\frac{q}{v}\right)^{L_{y}}(1+v)^{L_{y}}\right]^{q^{L_{y}}}
$$

\section{B. Eigenvalue for $d=L_{y}+1, \Lambda=G_{D}$}

For $d=L_{y}+1$, each factor of $V_{Z, G_{D}, L_{y}, d}$ in Eq. (7.4) reduces to a scalar $v$, and $H_{Z, G_{D}, L_{y}, d}$ and $\bar{H}_{Z, G_{D}, L_{y}, d}$ become one. Both of the transfer matrices $T_{Z, G_{D}, L_{y}, d}$ and $\bar{T}_{Z, G_{D}, L_{y}, d}$ for $d=L_{y}+1$ reduce to a scalar, namely $\lambda_{Z, G_{D}, L_{y}, L_{y}+1}=v^{L_{y}}$.

\section{Transfer Matrix for $d=L_{y}, \Lambda=G_{D}$}

The transfer matrix $T_{Z, G_{D}, L_{y}, L_{y}}$ has dimension $3 L_{y}+2$. We obtain the following general formulas:

$$
\begin{gathered}
\left(T_{Z, G_{D}, L_{y}, L_{y}}\right)_{1,1}=v^{L_{y}},\left(T_{Z, G_{D}, L_{y}, L_{y}}\right)_{2,2}=v^{L_{y}} w,\left(T_{Z, G_{D}, L_{y}, L_{y}}\right)_{2 L_{y}+1,2 L_{y}+1}=v^{L_{y}-1}(2 v+q-1) \\
\left(T_{Z, G_{D}, L_{y}, L_{y}}\right)_{2 j-1,2 j-1}=v^{L_{y}-1}(3 v+q-1) \quad \text { for } \quad L_{y} \geq 2 \text { and } 2 \leq j \leq L_{y} \\
\left(T_{Z, G_{D}, L_{y}, L_{y}}\right)_{2 j, 2 j}=v^{L_{y}-1} w(1+v) \quad \text { for } \quad 2 \leq j \leq L_{y}+1 \\
\left(T_{Z, G_{D}, L_{y}, L_{y}}\right)_{2 L_{y}+2,2 L_{y}+1}=v^{L_{y}-1}(v+q-1) \\
\left(T_{Z, G_{D}, L_{y}, L_{y}}\right)_{2 j, 2 j-1}=v^{L_{y}-1}(2 v+q-1) \quad \text { for } \quad L_{y} \geq 2 \text { and } 2 \leq j \leq L_{y} \\
\left(T_{Z, G_{D}, L_{y}, L_{y}}\right)_{2 j-1,2 j}=v^{L_{y}-1} w \quad \text { for } \quad 2 \leq j \leq L_{y}+1 \\
\left(T_{Z, G_{D}, L_{y}, L_{y}}\right)_{2 j-1,2 j+1}=\left(T_{Z, G_{D}, L_{y}, L_{y}-1}\right)_{2 j, 2 j+1}=v^{L_{y}} \quad \text { for } \quad 2 \leq j \leq L_{y} \\
\left(T_{Z, G_{D}, L_{y}, L_{y}}\right)_{2 j+1,2 j-1}=\left(T_{Z, G_{D}, L_{y}, L_{y}-1}\right)_{2 j+2,2 j-1}=v^{L_{y}} \quad \text { for } \quad 1 \leq j \leq L_{y} \\
\left(T_{Z, G_{D}, L_{y}, L_{y}}\right)_{j, j}=v^{L_{y}}(1+v) \quad \text { for } \quad 2 L_{y}+3 \leq j \leq 3 L_{y}+2 \\
=\left(T_{Z, G_{D}, L_{y}, L_{y}}\right)_{2 j+1,2 L_{y}+2+j}=\left(T_{Z, G_{D}, L_{y}, L_{y}-1}\right)_{2 j+2,2 L_{y}+2+j}=v^{L_{y}-1}(1+v) \quad \text { for } \quad 2 \leq j \leq L_{y} \\
\left(T_{Z, G_{D}, L_{y}, L_{y}}\right)_{3,2 L_{y}+3}=\left(T_{Z, G_{D}, L_{y}, L_{y}-1}\right)_{4,2 L_{y}+3}
\end{gathered}
$$




$$
\left(T_{Z, G_{D}, L_{y}, L_{y}}\right)_{2 L_{y}+2+j, 2 j-1}=\left(T_{Z, G_{D}, L_{y}, L_{y}-1}\right)_{2 L_{y}+2+j, 2 j+1}=v^{L_{y}+1} \quad \text { for } \quad 1 \leq j \leq L_{y}
$$

with all other elements equal to zero. $\bar{T}_{Z, G_{D}, L_{y}, L_{y}}$ is the same as $T_{Z, G_{D}, L_{y}, L_{y}}$ except for one element $\left(\bar{T}_{Z, G_{D}, L_{y}, L_{y}}\right)_{2,2}=v^{L_{y}}$.

We illustrate these general formulas for the cases $L_{y}=1$ and $L_{y}=2$ :

$$
\begin{aligned}
& T_{Z, G_{D}, 1,1}=\left(\begin{array}{ccccc}
v & 0 & 0 & 0 & 0 \\
0 & w v & 0 & 0 & 0 \\
v & 0 & x_{2} & w & v_{1} \\
v & 0 & x_{1} & w v_{1} & v_{1} \\
v^{2} & 0 & v^{2} & 0 & v v_{1}
\end{array}\right) \\
& T_{Z, G_{D}, 2,2}=v\left(\begin{array}{cccccccc}
v & 0 & 0 & 0 & 0 & 0 & 0 & 0 \\
0 & w v & 0 & 0 & 0 & 0 & 0 & 0 \\
v & 0 & x_{3} & w & v & 0 & v_{1} & v_{1} \\
v & 0 & x_{2} & w v_{1} & v & 0 & v_{1} & v_{1} \\
0 & 0 & v & 0 & x_{2} & w & 0 & v_{1} \\
0 & 0 & v & 0 & x_{1} & w v_{1} & 0 & v_{1} \\
v^{2} & 0 & v^{2} & 0 & 0 & 0 & v v_{1} & 0 \\
0 & 0 & v^{2} & 0 & v^{2} & 0 & 0 & v v_{1}
\end{array}\right)
\end{aligned}
$$

Thus, in general, the matrix $T_{Z, G_{D}, L_{y}, L_{y}}$ is almost the same as $T_{Z, s q, L_{y}+1, L_{y}}$ except for the first two rows. It is obvious that $T_{Z, G_{D}, L_{y}, L_{y}}$ always has one eigenvalue equal to $v^{L_{y}}$ and one equal to $v^{L_{y}} w$, and $\bar{T}_{Z, G_{D}, L_{y}, L_{y}}$ always has one eigenvalue equal to $v^{L_{y}}$ with multiplicity two.

As corollaries of our general result for $T_{Z, G_{D}, L_{y}, L_{y}}$ we calculate the determinant and trace:

$$
\begin{aligned}
\operatorname{det}\left(T_{Z, G_{D}, L_{y}, L_{y}}\right) & =\left(v^{L_{y}}\right)^{3 L_{y}+2}\left[w^{L_{y}+1}\left(1+\frac{q}{v}\right)^{L_{y}}(1+v)^{L_{y}}\right] \\
\operatorname{det}\left(\bar{T}_{Z, G_{D}, L_{y}, L_{y}}\right) & =\left(v^{L_{y}}\right)^{3 L_{y}+2}\left[w\left(1+\frac{q}{v}\right)(1+v)\right]^{L_{y}}
\end{aligned}
$$

which is the $d=L_{y}$ special case of (7.18), and

$$
\begin{aligned}
& \operatorname{Tr}\left(T_{Z, G_{D}, L_{y}, L_{y}}\right)=v^{L_{y}-1}\left[L_{y}\left(q-1+v^{2}+4 v\right)+w\left(v+L_{y}+v L_{y}\right)\right] \\
& \operatorname{Tr}\left(\bar{T}_{Z, G_{D}, L_{y}, L_{y}}\right)=v^{L_{y}-1}\left[L_{y}\left(q-1+v^{2}+4 v\right)+v+w L_{y}(1+v)\right]
\end{aligned}
$$

\section{SOME ILLUSTRATIVE CALCULATIONS}

\section{A. $L_{y}=1$}

We give some explicit results of the transfer matrices for cyclic strips in this section, beginning with the case $L_{y}=1$. We have exhibited the transfer matrix $T_{Z, s q, 1,0}$ in Eq. (4.6), and the quantity $T_{Z, s q, 1,1}=v$ has been given above. The Potts model partition function $Z\left(s q, L_{y} \times m, c y c ., q, v, w\right)$ was calculated for the the circuit graph in [43]. The results for eigenvalues agree. (The actual transfer matrices themselves are basis-dependent, and the basis used in [43] was different from ours, so the matrices are different, but the only part of the transfer matrices that enters into the partition function is the (powers of the) eigenvalues.) 


\section{B. Square-Lattice Strip, $L_{y}=2$}

The partition function for this case is given by the $L_{y}=2$ special case of Eq. (2.3). The transfer matrix for $d=0$ is

$$
T_{Z, s q, 2,0}=\left(\begin{array}{ccccc}
(q-1)^{2}+3(q-1) v+3 v^{2} & x_{1} w & x_{1} w & w^{2} v_{1} & x_{2} v_{1} \\
(q-1)^{2}+2(q-1) v+v^{2} & x_{1} w v_{1} & (q-1) w & w^{2} v_{1}^{2} & x_{1} v_{1} \\
(q-1)^{2}+2(q-1) v+v^{2} & (q-1) w & x_{1} v_{1} w & w^{2} v_{1}^{2} & x_{1} v_{1} \\
(q-1)^{2}+(q-1) v & (q-1) w v_{1} & (q-1) w v_{1} & w^{2} v_{1}^{3} & (q-1) v_{1} \\
v^{3} & 0 & 0 & 0 & v^{2} v_{1}
\end{array}\right)
$$

and the matrices $T_{Z, s q, 2,1}$ and $T_{Z, s q, 2,2}$ have been given above. The result for the cyclic case $Z(s q, 2 \times$ $m, c y c ., q, v, w)$ agrees with Ref. [44]. The partition function for the Möbius case follows from our general formulas also given above.

\section{Triangular-Lattice Strip, $L_{y}=2$}

We illustrate our results for the $L_{y}=2$ cyclic strip of the triangular lattice. We obtain

$$
T_{Z, \text { tri, }, 0}=\left(\begin{array}{ccccc}
(q-1)^{2}+4(q-1) v+5 v^{2}+v^{3} & x_{1} w & x_{2} w & w^{2} v_{1} & y_{3} v_{1} \\
(q-1)^{2}+3(q-1) v+3 v^{2}+v^{3} & x_{1} w v_{1} & x_{1} w & w^{2} v_{1}^{2} & y_{2} v_{1} \\
(q-1)^{2}+2(q-1) v+v^{2} & (q-1) w v_{1} & x_{1} v_{1} w & w^{2} v_{1}^{3} & x_{1} v_{1} \\
(q-1)^{2}+(q-1) v & (q-1) w v_{1}^{2} & (q-1) w v_{1} & w^{2} v_{1}^{4} & (q-1) v_{1} \\
v^{2} y_{3} & 0 & w v^{2} & 0 & v^{2} v_{1} v_{2}
\end{array}\right)
$$

The matrices $T_{Z, t r i, 2,1}$ and $T_{Z, t r i, 2,2}$ were given above.

\section{Honeycomb-lattice Strip, $L_{y}=2$}

For the $L_{y}=2$ cyclic strip of the honeycomb lattice we calculate

$$
T_{Z, h c, 2,0}=\left(\begin{array}{ccccc}
x_{1}^{2} & x_{1} w & x_{1} w & w^{2} & x_{2} \\
(q-1) x_{1} & x_{1} w v_{1} & (q-1) w & w^{2} v_{1} & x_{1} \\
(q-1) x_{1} & (q-1) w & x_{1} v_{1} w & w^{2} v_{1} & x_{1} \\
(q-1)^{2} & (q-1) w v_{1} & (q-1) w v_{1} & w^{2} v_{1}^{2} & q-1 \\
0 & 0 & 0 & 0 & v^{2}
\end{array}\right) T_{Z, s q, 2,0}
$$

where $T_{Z, s q, 2,0}$ is given in Eq. (8.1). The other matrices relevant for this strip were given above.

Acknowledgments: We thank F. Y. Wu for a valuable communication calling our attention to Ref. [15]. The research of R.S. was partially supported by the NSF grant PHY-00-98527. The research of S.C.C. was partially supported by the Taiwan NSC grant NSC-97-2112-M-006-007-MY3 and NSC-98-2119-M-002-001.

[1] R. B. Potts, Proc. Camb. Phil. Soc. 48, 106 (1952).

[2] F. Y. Wu, Rev. Mod. Phys. 54, 235 (1982).

[3] R. J. Baxter, Exactly Solved Models (Oxford Univ. Press., Oxford, UK, 1983).

[4] P. Martin, Potts Models and Related Problems in Statistical Mechanics (World Scientific, Singapore, 1991).

[5] F. Y. Wu, Exactly Solved Models: A Journey in Statistical Mechanics (World Scientific, Singapore, 2009). 
[6] D. J. A. Welsh, Complexity: Knots, Colourings, and Counting (Cambridge Univ. Press, Cambridge, 1993).

[7] N. Biggs, S.-C. Chang, F. M. Dong, B. Jackson, J. Jacobsen, G. Royle, R. Shrock, A. Sokal, C. Thomassen et al. 2008, in Workshop on Zeros of Graph Polynomials, Newton Institute for Mathematical Sciences, Cambridge University, http://www.newton.ac.uk/programmes/CSM/seminars

[8] L. Beaudin, J. Ellis-Monaghan, G. Pangborn, and R. Shrock, Discrete Math., in press (arXiv:0804.2468).

[9] J. Cardy, in C. Domb and J. Lebowitz, eds., Phase Transitions and Critical Phenomena (Academic Press, New York, 1987), v. 11, p. 55.

[10] P. Di Francesco, P. Mathieu, and D.Sénéchal, Conformal Field Theory (Springer, New York 1997).

[11] C. M. Fortuin and P. W. Kasteleyn, Physica 57, 536 (1972).

[12] W. T. Tutte, Canad. J. Math. 6, 301 (1954).

[13] N. Biggs, Algebraic Graph Theory (Cambridge Univ. Press, Cambridge, 1993).

[14] B. Bollobás, Modern Graph Theory (Springer, New York, 1998).

[15] F. Y. Wu, J. Stat. Phys. 18, 115 (1978).

[16] S.-Y. Kim and R. J. Creswick, Phys. Rev. E 58, 7006 (1998).

[17] S.-C. Chang and R. Shrock, arXiv:0907.0777.

[18] H. W. J. Blöte and M. P. Nightingale, Physica A 112, 405 (1982).

[19] H. Saleur, Nucl. Phys. B 360, 219 (1991).

[20] H. Saleur, Commun. Math. Phys. 132, 657 (1990).

[21] R. Shrock, Physica A 283, 388 (2000).

[22] J. Salas and A. Sokal, J. Stat. Phys. 104, 609 (2001).

[23] S.-C. Chang and R. Shrock, Physica A 296, 183 (2001).

[24] S.-C. Chang and R. Shrock, Physica A 296, 234 (2001).

[25] S.-C. Chang and R. Shrock, Physica A 286, 189 (2001).

[26] S.-C. Chang and R. Shrock, Int. J. Mod. Phys. B 15, 443 (2001).

[27] S.-C. Chang, J. Salas, and R. Shrock, J. Stat. Phys. 107, 1207 (2002).

[28] S.-C. Chang, J. Jacobsen, J. Salas, and R. Shrock, J. Stat. Phys. 114, 763 (2004).

[29] S.-C. Chang and R. Shrock, Physica A 347, 314 (2005).

[30] R. C. Read and W. T. Tutte, "Chromatic Polynomials", in Selected Topics in Graph Theory, 3, eds. L. W. Beineke and R. J. Wilson (Academic Press, New York, 1988), p. 15.

[31] S.-C. Chang and R. Shrock, Physica A 296, 131 (2001).

[32] S.-C. Chang and R. Shrock, Physica A 301, 301 (2001).

[33] S.-C. Chang and R. Shrock, Phys. Rev. E 64, 066116 (2001).

[34] M. Bernstein and N. J. A. Sloane, Linear Algebra Appl. 226, 57 (1995).

[35] N. J. A. Sloane, The On-Line Encyclopedia of Integer Sequences, http://www.research.att.com/ ${ }^{n j}$ as/sequences/ .

[36] S.-C. Chang and R. Shrock, Physica A 292, 307 (2001).

[37] N. L. Biggs, R. M. Damerell, and D. A. Sands, J. Combin. Theory B 12, 123 (1972).

[38] R. Shrock and S.-H. Tsai, J. Phys. A Letts. 32, L195 (1999).

[39] R. Shrock and S.-H. Tsai, Phys. Rev. E 60, 3512 (1999).

[40] R. Shrock, Phys. Lett. A 261, 57 (1999).

[41] S.-C. Chang and R. Shrock, Physica A 346, 400 (2005).

[42] N. Biggs and R. Shrock, J. Phys. A Lett. 32, L489 (1999).

[43] Z Glumac and K. Uzelac, J. Phys. A 27, 7709 (1994).

[44] B. Mirza and M. R. Bakhtiari, arXiv: cond-mat/0306007 